\title{
A Mini Library of Novel Triazolothiadiazepinylindole Analogues: Synthesis, Antioxidant and Antimicrobial Evaluations
}

\author{
Jaiprakash Sharanappa Biradar, ${ }^{1}$ Parveen Rajesab, ${ }^{1,2}$ \\ Naveen Jaiprakash Biradar, ${ }^{1}$ and Sasidhar Balappa Somappa ${ }^{1,3}$ \\ ${ }^{1}$ Central Research Lab, Department of Chemistry, Gulbarga University, Gulbarga, Karnataka State 585 106, India \\ ${ }^{2}$ Smt. V.G. Degree College for Women, Gulbarga, Karnataka State 585 102, India \\ ${ }^{3}$ Organic Chemistry Section, Chemical Science and Technology Division, National Institute for Interdisciplinary Science and \\ Technology (CSIR), Thiruvanthapuram-695 019, Kerala, India
}

Correspondence should be addressed to Jaiprakash Sharanappa Biradar; jsbiradar@yahoo.com

Received 23 August 2013; Accepted 4 December 2013; Published 20 February 2014

Academic Editors: L. Previtera and M. Wujec

Copyright (C) 2014 Jaiprakash Sharanappa Biradar et al. This is an open access article distributed under the Creative Commons Attribution License, which permits unrestricted use, distribution, and reproduction in any medium, provided the original work is properly cited.

\begin{abstract}
A new series of novel triazolothiadiazepinylindole analogues were synthesized with an aim to examine possible antioxidant and antimicrobial activities. The titled compounds $(3 \mathbf{a}-\mathbf{z})$ were obtained in good yield by reacting 5 - $(5$-substituted-3-phenyl- $1 \mathrm{H}$ indol-2-yl)-4-amino-4H-1,2,4-triazole-3-thiols 1a-c with 3-(2,5-disubstituted-1H-indol-3-yl)-1(4-substituted phenyl)prop-2-en-1ones $2 \mathbf{a}-\mathbf{i}$. All the newly synthesized compounds were characterized by IR, ${ }^{1} \mathrm{H}$ NMR, mass spectroscopic and analytical data. The synthesized analogues were tested for antioxidant and antimicrobial potency. Among the tested compounds $\mathbf{3 a - c}$ and $\mathbf{3 j} \mathbf{j}-\mathbf{l}$ have shown very promising free radical scavenging activity and total antioxidant capacity. Compounds $\mathbf{3 d}-\mathbf{f}, \mathbf{3 m}-\mathbf{o}$, and $\mathbf{3 s}-\mathbf{z}$ have shown excellent ferric reducing antioxidant activity. An outstanding antimicrobial activity is observed with compounds $\mathbf{3 a - c}$ and $3 \mathbf{j}-\mathbf{l}$.
\end{abstract}

\section{Introduction}

Antioxidants [1-3] act as "free radical scavengers" hence to prevent or slow the damage done by the free radicals [4-6]. Free-radical-induced oxidative stress associated with several cellular toxic processes including oxidative damage to protein, and DNA, membrane lipid oxidation, enzyme inactivation, and gene mutation leads to carcinogenesis [7]. Antioxidants are involved in processes such as immunity, protection against tissue damage, and reproduction and prevent growth or development caused by free radicals [810]. Antioxidants are useful in the prevention and treatment of Parkinson's and Alzheimer's disease [11-13].

Heterocycles constitute one of the major areas of organic chemistry and play important roles in drug discovery. Many of the best selling drugs currently in use contain one or more heterocyclic rings. Several fused heterocycles as well as biheterocycles are referred to as privileged structures [14]. Among them, sulfur- and nitrogen-containing heterocyclic compounds have maintained the interest of researchers and their unique structures led to several applications in different areas [15]. Triazoles and their derivatives constitute an important class of heterocyclic compounds and their analogues have been reported to possess various biological activities such as antimicrobial [16], anti-inflammatory [17], antihypertensive, anti-HIV [18], anticancer, and antitumor $[19,20]$. Several compounds containing 1,2,4-triazole rings known as drugs like fluconazole, posaconazole, alprazolam, [21] and triazolothiadiazepine analogues represent a wellknown class of drug substances at different stages of research, which possess antiviral [22] and antimicrobial properties [23].

Indole is a heterocycle of great importance in biological systems $[24,25]$. The indole moiety is present in a number 
of drugs currently [26] in the market; in our previous approaches, we have described some new indole analogues with highly potent antioxidant, DNA cleavage and antimicrobial activities [27-30].

Interestingly, we have developed a new green protocol for the synthesis of rapid and clean synthetic route towards mini library of triazolothiadiazepinylindole analogues, which showed in vitro antioxidant and antimicrobial activities.

\section{Materials and Methods}

2.1. Chemistry. All chemicals used in this investigation were of analytical grade and were purified whenever necessary. Melting points of the synthesized compounds were measured in open capillaries and are uncorrected. Reactions were monitored by thin-layer chromatography (TLC) on silica gel $60 \mathrm{~F}_{254}$ aluminium sheets (MERCK). Iodine vapour was used as detecting agent. IR spectra were recorded in $\mathrm{KBr}$ on PerkinElmer and FTIR spectrophotometer $\left(\nu_{\max }\right.$ in $\left.\mathrm{cm}^{-1}\right)$. ${ }^{1} \mathrm{H}$ NMR and ${ }^{13} \mathrm{C}$ NMR spectra on BRUKER AVENCE II $400-\mathrm{MHz}$ NMR spectrometer and the chemical shifts were expressed in ppm ( $\delta$ scale $)$ downfield from TMS as an internal reference. The mass spectra were recorded on LC-MSD-TrapSL instrument. The elemental analysis was performed by using FLASH EA 1112 SERIES instrument.

2.1.1. General Procedure for the Synthesis of Compounds $\mathbf{1 a}-\boldsymbol{c}$. The precursors 5-(5-substituted-3-phenyl-1H-indol-2-yl)-4amino-4H-1,2,4-triazole-3-thiols) (1a-c) were obtained from 3,5-disubstituted indol-2-carboxyhydrazides by reported method [31].

2.1.2. General Procedure for the Synthesis of Compounds $2 \boldsymbol{a}-\boldsymbol{i}$. 3-(2,5-disubstituted-1H-indol-3-yl)-1(4-substituted phenyl) prop-2-en-1-one $\mathbf{2 a - i}$ were prepared by reported method [29] by reacting disubstituted indole aldehydes with substituted acetophenone in the presence of piperidine in good yields.

\subsubsection{General Procedure for the Synthesis of Compounds $3 \boldsymbol{a}-\boldsymbol{z}$}

(1) Conventional Method. To a solution of substituted indolyltriazole $1 \mathbf{a}-\mathbf{c}(0.01 \mathrm{~mol})$ in acetic acid substituted chalcones $2 \mathbf{a}-\mathbf{i}(0.01 \mathrm{~mol})$ were added. The reaction mixture was refluxed 3-4 hrs. The completion of the reaction was monitored by TLC. After the completion, the reaction mixture was poured to a beaker containing $100 \mathrm{~mL}$ of ice-cold water. The crude products thus separated were filtered and recrystallized from ethanol to yield target compounds $3 \mathbf{a}-\mathbf{z}$.

(2) Microwave Oven Method. A mixture of substituted indolyl triazole $\mathbf{1 a}-\mathbf{c}(0.01 \mathrm{~mol})$ and substituted chalcones $\mathbf{2 a}-$ i $(0.01 \mathrm{~mol})$ was powdered, mixed, and introduced to borosil sample crucible containing few drops of acetic acid. This was subjected to microwave irradiation for 10 minutes with $70 \%$ microwave power. After the completion (TLC), reaction mixture was brought to room temperature, washed with ethanol, and recrystallized to get the title compounds $\mathbf{3 a}-\mathbf{z}$ which were found to be in good purity (TLC) and excellent yield.

8-(5-Chloro-2-phenyl-1H-indol-3-yl)-3-(5-chloro-3-phenyl-1H -indol-2-yl)-6-(4-chlorophenyl)-[1,2,4]triazolo[3,4-b][1,3,4] thiadiazepine (3a). IR ( $\mathrm{KBr}) v_{\max }\left(\mathrm{cm}^{-1}\right): 3180,3090,1654$, 1624, 1546; ${ }^{1} \mathrm{H}$ NMR (DMSO-d 6 - $\mathrm{CDCl}_{3}$ ) $\delta(\mathrm{ppm}): 12.47(\mathrm{~s}$, $1 \mathrm{H}$, indole $\mathrm{NH}), 11.63(\mathrm{~s}, 1 \mathrm{H}$, indole $\mathrm{NH}), 7.31-8.23(\mathrm{~m}, 20 \mathrm{H}$, Ar-H), 5.65 (s, $1 \mathrm{H},-\mathrm{CH}=) ;{ }^{13} \mathrm{C} \mathrm{NMR}\left(\mathrm{DMSO}-\mathrm{d}_{6}+\mathrm{CDCl}_{3}\right) \delta$ (ppm): 108, 111, 113, 117, 118, 118, 118, 120, 123, 125, 126, 126, 126, $128,128,128,128,129,129,129,130,132,133,134,135,138,138$, 144, 145, 166. MS: $m / z=712[\mathrm{M}]^{+\bullet}, 714[\mathrm{M}+2], 718[\mathrm{M}+4]$, $720[\mathrm{M}+6]$; Anal. calcd. for $\left(\mathrm{C}_{39} \mathrm{H}_{23} \mathrm{~N}_{6} \mathrm{Cl}_{3} \mathrm{~S}\right): \mathrm{C}, 65.60 ; \mathrm{H}$, 3.25 ; N, 11.77\%. Found: C, 65.59; H, 3.21; N, 11.75\%.

8-(5-Chloro-2-phenyl-1H-indol-3-yl)-3-(5-chloro-3-phenyl-1H -indol-2-yl)-6-phenyl-[1,2,4] triazolo[3,4-b][1,3,4] thiadiazepine $(3 \boldsymbol{b})$. IR $(\mathrm{KBr}) v_{\max }\left(\mathrm{cm}^{-1}\right): 3189,3049,1608,1579,1553$; ${ }^{1} \mathrm{H}$ NMR (DMSO-d ${ }_{6}+\mathrm{CDCl}_{3}$ ) $\delta$ (ppm): 11.15 (s, $1 \mathrm{H}$, indole $\mathrm{NH}), 10.25$ (s, 1H, indole NH), 7.29-8.72 (m, 21H, Ar-H), 4.95 (s, $1 \mathrm{H},-\mathrm{CH}=$ ); MS: $m / z=678[\mathrm{M}]^{+\bullet}, 680[\mathrm{M}+2], 682$ $[\mathrm{M}+4]$; Anal. calcd. for $\left(\mathrm{C}_{39} \mathrm{H}_{24} \mathrm{~N}_{6} \mathrm{Cl}_{2} \mathrm{~S}\right)$ : C, 68.92; $\mathrm{H}, 3.56$; N, 12.37\%. Found: C, 68.81; H, 3.52; N, 12.31\%.

8-(5-Chloro-2-phenyl-1H-indol-3-yl)-3-(5-chloro-3-phenyl-1H -indol-2-yl)-6-(4-methylphenyl[1,2,4] triazolo [3,4-b][1,3,4] thiadiazepine (3c). IR ( $\mathrm{KBr}) v_{\max }\left(\mathrm{cm}^{-1}\right): 3108,3053,1606$, 1574,$1553 ;{ }^{1} \mathrm{H}$ NMR (DMSO-d 6 - $\mathrm{CDCl}_{3}$ ) $\delta$ (ppm): 11.03 (s, $1 \mathrm{H}$, indole $\mathrm{NH}), 10.03$ (s, $1 \mathrm{H}$, indole $\mathrm{NH}), 7.29-8.14(\mathrm{~m}$, $20 \mathrm{H}, \mathrm{Ar}-\mathrm{H}), 5.35$ (s, $1 \mathrm{H},-\mathrm{CH}=), 2.44$ (s, 3H, $\mathrm{CH}_{3}$ ); MS: $m / z=692[\mathrm{M}]^{+\bullet}, 694[\mathrm{M}+2], 696[\mathrm{M}+4]$; Anal. calcd. for $\left(\mathrm{C}_{40} \mathrm{H}_{26} \mathrm{~N}_{6} \mathrm{Cl}_{2} \mathrm{~S}\right)$ : C, 69.26; $\mathrm{H}, 3.78 ; \mathrm{N}, 12.12 \%$. Found: $\mathrm{C}$, 69.15; H, 3.69; N, 12.21\%.

3-(5-Chloro-3-phenyl-1H-indol-2-yl)-6-(4-chlorophenyl)-8-(5methyl-2-phenyl-1H-indol-3-yl)-[1,2,4] triazolo[3,4-b][1,3,4] thiadiazepine $(3 \boldsymbol{d})$. IR ( $\mathrm{KBr}) v_{\max }\left(\mathrm{cm}^{-1}\right)$ : 3391, 3265, 1601, 1540, 1519; ${ }^{1} \mathrm{H}$ NMR (DMSO-d 6 - $\mathrm{CDCl}_{3}$ ) $\delta$ (ppm): 11.43 (s, $1 \mathrm{H}$, indole $\mathrm{NH}), 10.85$ (s, $1 \mathrm{H}$, indole $\mathrm{NH}), 6.40-9.13(\mathrm{~m}$, 20H, Ar-H), 4.91 (s, $1 \mathrm{H},-\mathrm{CH}=), 2.66$ (s, 3H, $\mathrm{CH}_{3}$ ); MS: $m / z=692[\mathrm{M}]^{+\bullet}, 694[\mathrm{M}+2], 696[\mathrm{M}+4]$; Anal. calcd. for $\left(\mathrm{C}_{40} \mathrm{H}_{26} \mathrm{~N}_{6} \mathrm{Cl}_{2} \mathrm{~S}\right)$ : C, 69.26; $\mathrm{H}, 3.78 ; \mathrm{N}, 12.12 \%$. Found: $\mathrm{C}$, 69.15; H, 3.69; N, 12.21\%.

3-(5-Chloro-3-phenyl-1H-indol-2-yl)-8-(5-methyl-2-phenyl$1 H$-indol-3-yl)-6-phenyl-[1,2,4]triazolo[3,4-b][1,3,4] thiadiazepine (3e). IR (KBr) $v_{\max }\left(\mathrm{cm}^{-1}\right): 3106,2996,1650,1590$, 1560; ${ }^{1} \mathrm{H}$ NMR (DMSO-d 6 - $\left.\mathrm{CDCl}_{3}\right) \delta$ (ppm): 11.01 (s, $1 \mathrm{H}$, indole $\mathrm{NH}), 10.12(\mathrm{~s}, 1 \mathrm{H}$, indole $\mathrm{NH}), 6.40-8.58(\mathrm{~m}, 21 \mathrm{H}$, Ar-H), 4.91 (s, $1 \mathrm{H},-\mathrm{CH}=), 2.55\left(\mathrm{~s}, 3 \mathrm{H}, \mathrm{CH}_{3}\right) ; \mathrm{MS}: m / z=658$ $[\mathrm{M}]^{+\bullet}, 660[\mathrm{M}+2]$; Anal. calcd. for $\left(\mathrm{C}_{40} \mathrm{H}_{27} \mathrm{~N}_{6} \mathrm{ClS}\right)$ : C, 72.88; $\mathrm{H}, 4.13$; N, 12.75\%. Found: C, 72.75; H, 4.09; N, 12.64\%.

3-(5-Chloro-3-phenyl-1H-indol-2-yl)-8-(5-methyl-2-phenyl$1 H$-indol-3-yl)-6-(4-methylphenyl)-[1,2,4] triazolo[3,4-b][1,3, 4]thiadiazepine (3f). IR (KBr) $v_{\max }\left(\mathrm{cm}^{-1}\right): 3443,3133,1602$, 1578, 1558; ${ }^{1} \mathrm{H}$ NMR (DMSO-d $\left.6+\mathrm{CDCl}_{3}\right) \delta$ (ppm): 11.31 (s, $1 \mathrm{H}$, indole $\mathrm{NH}), 10.25$ (s, $1 \mathrm{H}$, indole $\mathrm{NH}), 7.11-8.18(\mathrm{~m}, 20 \mathrm{H}$, Ar-H), 5.29 (s, 1H, $-\mathrm{CH}=), 2.54$ (s, 3H, $\left.\mathrm{CH}_{3}\right), 2.43$ (s, 3H, 
$\left.\mathrm{CH}_{3}\right)$; MS: $m / z=672[\mathrm{M}]^{+\bullet}, 674[\mathrm{M}+2]$; Anal. calcd. for $\left(\mathrm{C}_{41} \mathrm{H}_{29} \mathrm{~N}_{6} \mathrm{ClS}\right)$ : C, $73.15 ; \mathrm{H}, 4.34 ; \mathrm{N}, 12.48 \%$. Found: C, 73.02; $\mathrm{H}, 4.29 ; \mathrm{N}, 12.37 \%$.

3-(5-Chloro-3-phenyl-1H-indol-2-yl)-6-(4-chlorophenyl)-8(1H-indol-3-yl)-[1,2,4] triazolo[3,4-b][1,3,4]thiadiazepine (3g). IR $(\mathrm{KBr}) v_{\max }\left(\mathrm{cm}^{-1}\right): 3239,3098,1607,1578,1553 ;{ }^{1} \mathrm{H}$ NMR $\left(\mathrm{DMSO}-\mathrm{d}_{6}+\mathrm{CDCl}_{3}\right) \delta(\mathrm{ppm}): 11.78(\mathrm{~s}, 1 \mathrm{H}$, indole $\mathrm{NH}), 10.51$ (s, $1 \mathrm{H}$, indole $\mathrm{NH}), 6.40-8.56(\mathrm{~m}, 17 \mathrm{H}, \mathrm{Ar}-\mathrm{H}), 4.94(\mathrm{~s}, 1 \mathrm{H},-$ $\mathrm{CH}=)$; MS: $m / z=602[\mathrm{M}]^{+\bullet}, 604[\mathrm{M}+2], 606[\mathrm{M}+4]$; Anal. calcd. for $\left(\mathrm{C}_{33} \mathrm{H}_{20} \mathrm{~N}_{6} \mathrm{Cl}_{2} \mathrm{~S}\right)$ : C, 65.67; H, 3.34; N, 13.92; Found: C, $65.57 ; \mathrm{H}, 3.28 ; \mathrm{N}, 13.85 \%$.

3-(5-Chloro-3-phenyl-1H-indol-2-yl)-8-(1H-indol-3-yl)-6phenyl-[1,2,4] triazolo [3,4-b][1,3,4] thiadiazepine (3h). IR $(\mathrm{KBr}) \nu_{\max }\left(\mathrm{cm}^{-1}\right): 3404,3104,1608,1558,1505 ;{ }^{1} \mathrm{H} \mathrm{NMR}$ $\left(\mathrm{DMSO}-\mathrm{d}_{6}+\mathrm{CDCl}_{3}\right) \delta(\mathrm{ppm}): 10.61(\mathrm{~s}, 1 \mathrm{H}$, indole $\mathrm{NH}), 10.01$ (s, 1H, indole $\mathrm{NH}), 6.43-8.91(\mathrm{~m}, 18 \mathrm{H}, \mathrm{Ar}-\mathrm{H}), 5.15$ (s, $1 \mathrm{H}$, $-\mathrm{CH}=)$; MS: $m / z=568[\mathrm{M}]^{+\bullet}, 570[\mathrm{M}+2]$; Anal. calcd. for $\left(\mathrm{C}_{33} \mathrm{H}_{21} \mathrm{~N}_{6} \mathrm{ClS}\right): \mathrm{C}, 69.65 ; \mathrm{H}, 3.72 ; \mathrm{N}, 14.77 \%$. Found: C, 69.55; $\mathrm{H}, 3.65 ; \mathrm{N}, 14.71 \%$.

3-(5-Chloro-3-phenyl-1H-indol-2-yl)-8-(1H-indol-3-yl)-6-(4methylphenyl)-[1,2,4]triazolo[3,4-b][1,3,4] thiadiazepine (3i). IR $(\mathrm{KBr}) v_{\max }\left(\mathrm{cm}^{-1}\right): 3160,3096,1645,1603 ;{ }^{1} \mathrm{H} \mathrm{NMR}$ $\left(\mathrm{DMSO}-\mathrm{d}_{6}+\mathrm{CDCl}_{3}\right) \delta(\mathrm{ppm}): 11.97(\mathrm{~s}, 1 \mathrm{H}$, indole $\mathrm{NH}), 11.39$ (s, 1H, indole NH), 6.80-7.85 (m, 17H, Ar-H), 5.59 (s, 1H, $-\mathrm{CH}=$ ), 2.64 (s, 3H, $\mathrm{CH}_{3}$ ); MS: $m / z=582[\mathrm{M}]^{+\bullet}, 584[\mathrm{M}+2]$; Anal. calcd. for $\left(\mathrm{C}_{34} \mathrm{H}_{23} \mathrm{~N}_{6} \mathrm{ClS}\right)$ : C, 70.03; H, 3.98; N, $14.41 \%$. Found: C, 69.91; H, 3.95; N, 14.31\%.

3-(5-Bromo-3-phenyl-1H-indol-2-yl)-8-(5-chloro-2-phenyl$1 H$-indol-3-yl)-6-(4-chlorophenyl)-[1,2,4]triazolo[3,4-b][1,3, 4]thiadiazepine (3j). IR (KBr) $\nu_{\max }\left(\mathrm{cm}^{-1}\right)$ : 3148, 3098, 1643, 1589, 1551; ${ }^{1} \mathrm{H}$ NMR (DMSO-d $\left.{ }_{6}+\mathrm{CDCl}_{3}\right) \delta(\mathrm{ppm}): 12.48(\mathrm{~s}$, $1 \mathrm{H}$, indole $\mathrm{NH}), 11.99$ (s, $1 \mathrm{H}$, indole $\mathrm{NH}), 7.07-8.23(\mathrm{~m}, 20 \mathrm{H}$, Ar-H), 5.60 (s, $1 \mathrm{H},-\mathrm{CH}=)$; MS: $m / z=756[\mathrm{M}]^{+\bullet}, 758[\mathrm{M}+2]$, $760[\mathrm{M}+4], 762[\mathrm{M}+6]$; Anal. calcd. for $\left(\mathrm{C}_{39} \mathrm{H}_{23} \mathrm{~N}_{6} \mathrm{BrCl}_{2} \mathrm{~S}\right)$ : C, 61.75; H, 3.06; N, 11.08\%. Found: C, 61.69; H, 3.01; N, $10.91 \%$.

3-(5-Bromo-3-phenyl-1H-indol-2-yl)-8-(5-chloro-2-phenyl$1 H$-indol-3-yl)-6-phenyl-[1,2,4]triazolo[3,4-b][1,3,4] thiadiazepine (3k). IR (KBr) $v_{\max }\left(\mathrm{cm}^{-1}\right): 3158,3068,1590,1576$, 1551; ${ }^{1} \mathrm{H}$ NMR (DMSO-d 6 - $\left.\mathrm{CDCl}_{3}\right) \delta$ (ppm): 11.15 (s, $1 \mathrm{H}$, indole $\mathrm{NH}), 10.05(\mathrm{~s}, 1 \mathrm{H}$, indole $\mathrm{NH}), 7.29-8.72(\mathrm{~m}, 21 \mathrm{H}$, Ar-H), 5.45 (s, $1 \mathrm{H},-\mathrm{CH}=)$; MS: $m / z=722[\mathrm{M}]^{+\bullet}, 724[\mathrm{M}+2]$, $726[\mathrm{M}+4]$; Anal. calcd. for $\left(\mathrm{C}_{39} \mathrm{H}_{24} \mathrm{~N}_{6} \mathrm{BrClS}\right): \mathrm{C}, 64.69 ; \mathrm{H}$, $3.34 ;$ N, 11.61\%. Found: C, 65.21; H, 3.51; N, 11.45\%.

3-(5-Bromo-3-phenyl-1H-indol-2-yl)-8-(5-chloro-2-phenyl$1 H$-indol-3-yl)-6-(4-methylphenyl)-[1,2,4] triazolo[3,4-b][1,3, 4] thiadiazepine (3l). IR (KBr) $\nu_{\max }\left(\mathrm{cm}^{-1}\right): 3108,3029,1644$, 1606, 1553; ${ }^{1} \mathrm{H}$ NMR (DMSO- $\left.\mathrm{d}_{6}+\mathrm{CDCl}_{3}\right) \delta$ (ppm): 11.03 (s, $1 \mathrm{H}$, indole $\mathrm{NH}), 10.33$ (s, $1 \mathrm{H}$, indole $\mathrm{NH}), 7.2-8.1(\mathrm{~m}$, 20H, Ar-H), 5.05 (s, 1H, $-\mathrm{CH}=), 2.44$ (s, 3H, $\left.\mathrm{CH}_{3}\right)$; MS: $m / z=736[\mathrm{M}]^{+\bullet}, 738[\mathrm{M}+2], 740[\mathrm{M}+4]$; Anal. calcd. for $\left(\mathrm{C}_{40} \mathrm{H}_{26} \mathrm{~N}_{6} \mathrm{BrClS}\right): \mathrm{C}, 65.09 ; \mathrm{H}, 3.55 ; \mathrm{N}, 11.39 \%$. Found: $\mathrm{C}$, 64.89; H, 3.51; N, 11.28\%.
3-(5-Bromo-3-phenyl-1H-indol-2-yl)-6-(4-chlorophenyl)-8(5-methyl-2-phenyl-1H-indol-3-yl)-[1,2,4]triazolo[3,4-b][1,3, 4] thiadiazepine $(3 \mathrm{~m})$. IR $(\mathrm{KBr}) \nu_{\max }\left(\mathrm{cm}^{-1}\right): 3176,3048,1623$, 1584, 1509; ${ }^{1} \mathrm{H}$ NMR (DMSO-d ${ }_{6}+\mathrm{CDCl}_{3}$ ) $\delta$ (ppm): 12.20 (s, $1 \mathrm{H}$, indole $\mathrm{NH}), 11.15(\mathrm{~s}, 1 \mathrm{H}$, indole $\mathrm{NH}), 6.32-8.13(\mathrm{~m}$, $20 \mathrm{H}, \mathrm{Ar}-\mathrm{H}), 5.60$ (s, $1 \mathrm{H},-\mathrm{CH}=), 1.74$ (s, 3H, $\left.\mathrm{CH}_{3}\right) ; \mathrm{MS}$ : $m / z=736[\mathrm{M}]^{+\bullet}, 738[\mathrm{M}+2], 740[\mathrm{M}+4]$; Anal. calcd. for $\left(\mathrm{C}_{40} \mathrm{H}_{26} \mathrm{~N}_{6} \mathrm{BrClS}\right): \mathrm{C}, 65.09 ; \mathrm{H}, 3.55 ; \mathrm{N}, 11.39 \%$. Found: $\mathrm{C}$, 64.09; H, 3.51; N, 11.28\%.

3-(5-Bromo-3-phenyl-1H-indol-2-yl)-8-(5-methyl-2-phenyl$1 H$-indol-3-yl)-6-phenyl-[1,2,4]triazolo[3,4-b][1,3,4]thiadiazepine (3n). IR (KBr) $v_{\max }\left(\mathrm{cm}^{-1}\right): 3240,3198,1604,1558$, 1553; ${ }^{1} \mathrm{H}$ NMR (DMSO-d $\left.6+\mathrm{CDCl}_{3}\right) \delta$ (ppm): 11.01 (s, $1 \mathrm{H}$, indole $\mathrm{NH}), 9.90(\mathrm{~s}, 1 \mathrm{H}$, indole $\mathrm{NH}), 6.40-8.58(\mathrm{~m}, 21 \mathrm{H}$, Ar-H), 4.31 (s, $1 \mathrm{H},-\mathrm{CH}=), 2.55$ (s, 3H, $\left.\mathrm{CH}_{3}\right)$; MS: $m / z=702$ $[\mathrm{M}]^{+\bullet}, 704[\mathrm{M}+2]$; Anal. calcd. for $\left(\mathrm{C}_{40} \mathrm{H}_{27} \mathrm{~N}_{6} \mathrm{BrS}\right)$ : C, 68.28; $\mathrm{H}, 3.87$; N, 11.94\%. Found: C, 68.18; H, 3.82; N, 11.83\%.

3-(5-bromo-3-phenyl-1H-indol-2-yl)-8-(5-methyl-2-phenyl$1 H$-indol-3-yl)-6-(4-methylphenyl)-[1,2,4]triazolo[3,4-b][1,3, 4] thiadiazepine (3o). IR ( $\mathrm{KBr}) \nu_{\max }\left(\mathrm{cm}^{-1}\right): 3117,3047,1641$, 1606, 1573; ${ }^{1} \mathrm{H}$ NMR (DMSO- $\mathrm{d}_{6}+\mathrm{CDCl}_{3}$ ) $\delta$ (ppm): 10.25 (s, $1 \mathrm{H}$, indole $\mathrm{NH}), 9.95(\mathrm{~s}, 1 \mathrm{H}$, indole $\mathrm{NH}), 7.11-8.18(\mathrm{~m}$, 20H, Ar-H), 5.15 (s, 1H, $-\mathrm{CH}=), 2.54$ (s, 3H, $\mathrm{CH}_{3}$ ), 2.43 (s, $\left.3 \mathrm{H}, \mathrm{CH}_{3}\right)$; MS: $m / z=716[\mathrm{M}]^{+\bullet}, 718[\mathrm{M}+2]$; Anal. calcd. for $\left(\mathrm{C}_{41} \mathrm{H}_{29} \mathrm{~N}_{6} \mathrm{BrS}\right)$ : C, 68.62; H, 4.07; N, 11.71\%. Found: C, 68.52; $\mathrm{H}, 4.05 ; \mathrm{N}, 11.59 \%$.

3-(5-Bromo-3-phenyl-1H-indol-2-yl)-6-(4-chlorophenyl)-8(1H-indol-3-yl)-[1,2,4] triazolo[3,4-b] [1,3,4] thiadiazepine (3p). IR (KBr) $v_{\max }\left(\mathrm{cm}^{-1}\right): 3167,3047,1648,1589,1558 ;{ }^{1} \mathrm{H}$ NMR (DMSO- $\left.\mathrm{d}_{6}+\mathrm{CDCl}_{3}\right) \delta$ (ppm): 10.61 (s, $1 \mathrm{H}$, indole $\mathrm{NH}), 10.23$ (s, 1H, indole NH), 6.83-8.19 (m, 17H, Ar-H), 5.19 (s, 1H, -CH=); MS: $m / z=646[\mathrm{M}]^{+\bullet}, 648[\mathrm{M}+2], 650$ $[\mathrm{M}+4]$; Anal. calcd. for $\left(\mathrm{C}_{33} \mathrm{H}_{20} \mathrm{~N}_{6} \mathrm{BrClS}\right)$ : C, 61.17; H, 3.11; N, 12.97\%. Found: C, 61.12; H, 3.09; N, 12.85\%.

3-(5-Bromo-3-phenyl-1H-indol-2-yl)-8-(1H-indol-3-yl)-6-phenyl-[1,2,4]triazolo[3,4-b][1,3,4] thiadiazepine (3q). IR ( $\mathrm{KBr})$ $\nu_{\max }\left(\mathrm{cm}^{-1}\right): 3097,2998,1606,1578,1551 ;{ }^{1} \mathrm{H}$ NMR (DMSO$\left.\mathrm{d}_{6}+\mathrm{CDCl}_{3}\right) \delta(\mathrm{ppm}): 11.01(\mathrm{~s}, 1 \mathrm{H}$, indole $\mathrm{NH}), 10.01(\mathrm{~s}$, $1 \mathrm{H}$, indole $\mathrm{NH}), 6.83-8.91(\mathrm{~m}, 18 \mathrm{H}, \mathrm{Ar}-\mathrm{H}), 5.15(\mathrm{~s}, 1 \mathrm{H}$, $-\mathrm{CH}=)$; MS: $m / z=612[\mathrm{M}]^{+\bullet}, 614[\mathrm{M}+2]$; Anal. calcd. For $\left(\mathrm{C}_{33} \mathrm{H}_{21} \mathrm{~N}_{6} \mathrm{BrS}\right)$ : C, 64.60; H, 3.45; N, 13.70\%. Found: C, 64.56; H, 3.41; N, 13.51\%.

3-(5-Bromo-3-phenyl-1H-indol-2-yl)-8-(1H-indol-3-yl)-6-(4methylphenyl)-[1,2,4] triazolo[3,4-b][1,3,4] thiadiazepine (3r). IR $(\mathrm{KBr}) \nu_{\max }\left(\mathrm{cm}^{-1}\right): 3104,3049,1608,1598,1558 ;{ }^{1} \mathrm{H}$ NMR $\left(\mathrm{DMSO}-\mathrm{d}_{6}+\mathrm{CDCl}_{3}\right) \delta$ (ppm): 11.07 (s, 1H, indole NH), 10.19 (s, $1 \mathrm{H}$, indole $\mathrm{NH}), 6.80-7.85(\mathrm{~m}, 17 \mathrm{H}, \mathrm{Ar}-\mathrm{H}), 5.39(\mathrm{~s}, 1 \mathrm{H}$, $-\mathrm{CH}=) 2.64\left(\mathrm{~s}, 3 \mathrm{H}, \mathrm{CH}_{3}\right)$; MS: $m / z=626[\mathrm{M}]^{+\bullet}, 628[\mathrm{M}+2]$; Anal. calcd. for $\left(\mathrm{C}_{34} \mathrm{H}_{23} \mathrm{~N}_{6} \mathrm{BrS}\right)$ : C, 65.07; $\mathrm{H}, 3.69 ; \mathrm{N}, 13.39 \%$. Found: C, 64.95; H, 3.65; N, 13.28\%.

8-(5-Chloro-2-phenyl-1H-indol-3-yl)-6-(4-chlorophenyl)-3-(5methyl-3-phenyl-1H-indol-2-yl)-[1,2,4]triazolo[3,4-b][1,3,4] thiadiazepine (3s). IR (KBr) $v_{\max }\left(\mathrm{cm}^{-1}\right): 3219,3196,1641$, 
1589, 1552; ${ }^{1} \mathrm{H}$ NMR (DMSO-d $\left.{ }_{6}+\mathrm{CDCl}_{3}\right) \delta$ (ppm): 11.01 $(\mathrm{s}, 1 \mathrm{H}$, indole $\mathrm{NH}), 10.25(\mathrm{~s}, 1 \mathrm{H}$, indole $\mathrm{NH}), 6.40-8.59(\mathrm{~m}$, 20H, Ar-H), 4.95 (s, $1 \mathrm{H},-\mathrm{CH}=), 2.56$ (s, 3H, $\mathrm{CH}_{3}$ ); MS: $m / z=692[\mathrm{M}]^{+\bullet}, 694[\mathrm{M}+2], 696[\mathrm{M}+4]$; Anal. calcd. for $\left(\mathrm{C}_{40} \mathrm{H}_{26} \mathrm{~N}_{6} \mathrm{Cl}_{2} \mathrm{~S}\right)$ : C, 69.26; $\mathrm{H}, 3.78 ; \mathrm{N}, 12.12 \%$. Found: $\mathrm{C}$, $69.14 ; \mathrm{H}, 3.72 ; \mathrm{N}, 12.02 \%$.

8-(5-Chloro-2-phenyl-1H-indol-3-yl)-3-(5-methyl-3-phenyl$1 H$-indol-2-yl)-6-phenyl-[1,2,4]triazolo[3,4-b][1,3,4] thiadiazepine (3t). IR (KBr) $v_{\max }\left(\mathrm{cm}^{-1}\right)$ : 3244, 3189, 1641, 1604, 1552; ${ }^{1} \mathrm{H}$ NMR (DMSO- $\mathrm{d}_{6}+\mathrm{CDCl}_{3}$ ) $\delta$ (ppm): 12.39 (s, $1 \mathrm{H}$, indole $\mathrm{NH}), 11.09$ (s, 1H, indole NH), 6.80-7.85 (m, 21H, Ar-H), 5.15 (s, $1 \mathrm{H},-\mathrm{CH}=), 2.76\left(\mathrm{~s}, 3 \mathrm{H}, \mathrm{CH}_{3}\right) ; \mathrm{MS}: m / z=658[\mathrm{M}]^{+\bullet}, 660$ $[\mathrm{M}+2]$; Anal. calcd. for $\left(\mathrm{C}_{40} \mathrm{H}_{27} \mathrm{~N}_{6} \mathrm{ClS}\right): \mathrm{C}, 72.88 ; \mathrm{H}, 4.13 ; \mathrm{N}$, $12.75 \%$. Found: C, 72.78; H, 4.10; N, 12.59\%.

8-(5-Chloro-2-phenyl-1H-indol-3-yl)-3-(5-methyl-3-phenyl$1 H$-indol-2-yl)-6-(4-methylphenyl)-[1,2,4]triazolo[3,4-b][1,3, 4] thiadiazepine $(3 \boldsymbol{u})$. IR (KBr) $\nu_{\max }\left(\mathrm{cm}^{-1}\right): 3248,3198,1606$, 1579, 1552; ${ }^{1} \mathrm{H}$ NMR (DMSO-d $6+\mathrm{CDCl}_{3}$ ) $\delta$ (ppm): 12.20 (s, $1 \mathrm{H}$, indole $\mathrm{NH}), 11.98(\mathrm{~s}, 1 \mathrm{H}$, indole $\mathrm{NH}), 7.05-8.13(\mathrm{~m}$, $20 \mathrm{H}, \mathrm{Ar}-\mathrm{H}), 4.37$ (s, 1H, $-\mathrm{CH}=), 2.57$ (s, 3H, $\left.\mathrm{CH}_{3}\right), 2.01$ (s, $\left.3 \mathrm{H}, \mathrm{CH}_{3}\right)$; MS: $m / z=672[\mathrm{M}]^{+\bullet}, 674[\mathrm{M}+2]$; Anal. calcd. for $\left(\mathrm{C}_{41} \mathrm{H}_{29} \mathrm{~N}_{6} \mathrm{ClS}\right)$ : C, 73.15; H, 4.34; N, 12.48\%. Found: C, 73.28; $\mathrm{H}, 4.31 ; \mathrm{N}, 12.36 \%$.

6-(4-Chlorophenyl)-8-(5-methyl-2-phenyl-1H-indol-3-yl)-3(5-methyl-3-phenyl-1H-indol-2-yl)-[1,2,4] triazolo[3,4-b][1,3, 4] thiadiazepine $(3 v)$. IR $(\mathrm{KBr}) v_{\max }\left(\mathrm{cm}^{-1}\right): 3248,3198,1604$, 1574, 1552; ${ }^{1} \mathrm{H}$ NMR (DMSO-d 6 - $\left.\mathrm{CDCl}_{3}\right) \delta$ (ppm): 11.39 (s, $1 \mathrm{H}$, indole $\mathrm{NH}), 10.39$ (s, $1 \mathrm{H}$, indole $\mathrm{NH}), 6.43-8.91(\mathrm{~m}, 20 \mathrm{H}$, Ar-H), 4.55 (s, $1 \mathrm{H},-\mathrm{CH}=), 2.58\left(\mathrm{~s}, 3 \mathrm{H}, \mathrm{CH}_{3}\right) ; \mathrm{MS}: m / z=672$ $[\mathrm{M}]^{+\bullet}, 674[\mathrm{M}+2]$; Anal. calcd. for $\left(\mathrm{C}_{41} \mathrm{H}_{29} \mathrm{~N}_{6} \mathrm{ClS}\right)$ : C, 73.15; $\mathrm{H}, 4.34 ; \mathrm{N}, 12.48 \%$. Found: $\mathrm{C}, 73.28 ; \mathrm{H}, 4.31 ; \mathrm{N}, 12.36 \%$.

8-(5-Methyl-2-phenyl-1H-indol-3-yl)-3-(5-methyl-3-phenyl$1 H$-indol-2-yl)-6-phenyl-[1,2,4]triazolo[3,4-b][1,3,4]thiadiazepine $(3 w)$. IR $(\mathrm{KBr}) v_{\max }\left(\mathrm{cm}^{-1}\right): 3248,3179,1604,1574$, 1556; ${ }^{1} \mathrm{H}$ NMR (DMSO-d $\left.6+\mathrm{CDCl}_{3}\right) \delta$ (ppm): 11.05 (s, $1 \mathrm{H}$, indole $\mathrm{NH}), 10.10(\mathrm{~s}, 1 \mathrm{H}$, indole $\mathrm{NH}), 6.32-8.13(\mathrm{~m}, 21 \mathrm{H}$, Ar-H), 5.60 (s, $1 \mathrm{H},-\mathrm{CH}=), 2.23\left(\mathrm{~s}, 6 \mathrm{H}, \mathrm{CH}_{3}\right) ; \mathrm{MS}: m / z=638$ $[\mathrm{M}]^{+\bullet}$; Anal. calcd. For $\left(\mathrm{C}_{41} \mathrm{H}_{30} \mathrm{~N}_{6} \mathrm{~S}\right): \mathrm{C}, 77.09 ; \mathrm{H}, 4.73 ; \mathrm{N}$, 13.16\%. Found: C, 77.06; H, 4.68; N, 13.03\%.

8-(5-Methyl-2-phenyl-1H-indol-3-yl)-3-(5-methyl-3-phenyl$1 H$-indol-2-yl)-6-(4-methylphenyl)-[1,2,4]triazolo[3,4-b][1,3, 4] thiadiazepine $(3 \boldsymbol{x})$. IR (KBr) $\nu_{\max }\left(\mathrm{cm}^{-1}\right): 3184,3148,1606$, 1574, 1553; ${ }^{1} \mathrm{H}$ NMR (DMSO-d 6 - $\left.\mathrm{CDCl}_{3}\right) \delta$ (ppm): 11.05 (s, $1 \mathrm{H}$, indole $\mathrm{NH}), 10.07$ (s, $1 \mathrm{H}$, indole $\mathrm{NH}), 6.40-8.77(\mathrm{~m}, 20 \mathrm{H}$, Ar-H), 4.15 (s, $1 \mathrm{H},-\mathrm{CH}=), 2.54\left(\mathrm{~s}, 6 \mathrm{H}, \mathrm{CH}_{3}\right), 2.31(\mathrm{~s}, 3 \mathrm{H}$, $\left.\mathrm{CH}_{3}\right)$; MS: $m / z=652[\mathrm{M}]^{+\bullet}$; Anal. calcd. for $\left(\mathrm{C}_{42} \mathrm{H}_{32} \mathrm{~N}_{6} \mathrm{~S}\right): \mathrm{C}$, 77.27; H, 4.94; N, 12.87\%. Found: C, 77.17; H, 4.91; N, $12.96 \%$.

8-(1H-Indol-3-yl)-3-(5-methyl-3-phenyl-1H-indol-2-yl)-6phenyl-[1,2,4] triazolo[3,4-b][1,3,4] thiadiazepine (3y). IR $(\mathrm{KBr}) \nu_{\max }\left(\mathrm{cm}^{-1}\right): 3354,3258,1674,1595,1554 ;{ }^{1} \mathrm{H} \mathrm{NMR}$ $\left(\mathrm{DMSO}_{-} \mathrm{d}_{6}+\mathrm{CDCl}_{3}\right) \delta(\mathrm{ppm}): 10.61(\mathrm{~s}, 1 \mathrm{H}$, indole $\mathrm{NH}), 10.01$ (s, $1 \mathrm{H}$, indole $\mathrm{NH}), 6.40-8.91(\mathrm{~m}, 18 \mathrm{H}, \mathrm{Ar}-\mathrm{H}), 4.85(\mathrm{~s}, 1 \mathrm{H}$, $-\mathrm{CH}=), 2.54\left(\mathrm{~s}, 3 \mathrm{H}, \mathrm{CH}_{3}\right)$; MS: $m / z=548[\mathrm{M}]^{+\bullet}$; Anal. calcd. for $\left(\mathrm{C}_{34} \mathrm{H}_{24} \mathrm{~N}_{6} \mathrm{~S}\right)$ : C, 74.43; H, 4.41; N, 15.32\%. Found: C, 74.39; H, 4.39; N, 15.25\%.

6-(4-Chlorophenyl)-8-(1H-indol-3-yl)-3-(5-methyl-3-phenyl$1 H$-indol-2-yl)-[1,2,4]triazolo[3,4-b][1,3,4] thiadiazepine (3z). IR $(\mathrm{KBr}) \nu_{\max }\left(\mathrm{cm}^{-1}\right): 3391,3244,1667,1601,1540 ;{ }^{1} \mathrm{H}$ NMR $\left(\mathrm{DMSO}_{-} \mathrm{d}_{6}+\mathrm{CDCl}_{3}\right) \delta(\mathrm{ppm}): 12.23(\mathrm{~s}, 1 \mathrm{H}$, indole $\mathrm{NH}), 10.11$ (s, $1 \mathrm{H}$, indole $\mathrm{NH}), 6.76-7.61(\mathrm{~m}, 17 \mathrm{H}, \mathrm{Ar}-\mathrm{H}), 4.37 \mathrm{~s}, 1 \mathrm{H}$, $-\mathrm{CH}=), 2.08\left(\mathrm{~s}, 3 \mathrm{H}, \mathrm{CH}_{3}\right)$; MS: $m / z=582[\mathrm{M}]^{+\bullet} 584[\mathrm{M}+2]$; Anal. calcd. For $\left(\mathrm{C}_{34} \mathrm{H}_{25} \mathrm{~N}_{6} \mathrm{~S}\right)$ : C, 70.03; H, 3.98; N, 14.41\%. Found: C, 69.98; H, 3.95; N, 14.35\%.

\subsection{Biological Activities}

\subsubsection{Antioxidant Activities}

(1) Free Radical Scavenging Activity. Free radical scavenging activity was done by DPPH method [32]. Different concentrations $(25 \mu \mathrm{g}, 50 \mu \mathrm{g}$, and $100 \mu \mathrm{g})$ of samples and butylated hydroxy anisole (BHA) were taken in different test tubes. The volume was adjusted to $100 \mu \mathrm{L}$ by adding $\mathrm{MeOH}$. Five milliliters of $0.1 \mathrm{mM}$ methanolic solution of DPPH was added to these tubes and shaken vigorously. The tubes were allowed to stand at $27^{\circ} \mathrm{C}$ for $20 \mathrm{~min}$. The control was prepared as above without any samples. The absorbances of samples were measured at $517 \mathrm{~nm}$. Radical scavenging activity was calculated using the following formula:

$\%$ Radical scavenging activity

$$
=\left[\frac{(\text { Control OD }- \text { Sample OD })}{(\text { Control OD })}\right] \times 100 \text {. }
$$

(2) Total Antioxidant Capacity. Various concentrations of samples $(25 \mu \mathrm{g}, 50 \mu \mathrm{g}$, and $100 \mu \mathrm{g})$ were taken in a series of test tubes. To this, $1.9 \mathrm{~mL}$ of reagent solution $(0.6 \mathrm{M}$ sulfuric acid, $28 \mathrm{mM}$ sodium phosphate, and $4 \mathrm{mM}$ ammonium molybdate) was added. The tubes were incubated at $95^{\circ} \mathrm{C}$ for $90 \mathrm{~min}$ and allowed to cool. The absorbance of each aqueous solution was measured at $695 \mathrm{~nm}$ against a blank. Antioxidant capacities are expressed as equivalents of ascorbic acid. Ascorbic acid equivalents were calculated using standard graph of ascorbic acid. The values are expressed as ascorbic acid equivalents in $\mu \mathrm{g}$ per mg of samples.

(3) Ferric Reducing Antioxidant Power. Various concentrations of samples $(25 \mu \mathrm{g}, 50 \mu \mathrm{g}$, and $100 \mu \mathrm{g})$ were mixed with $2.5 \mathrm{~mL}$ of $200 \mathrm{mmol} / \mathrm{L}$ sodium phosphate buffer $(\mathrm{pH} 6.6)$ and $2.5 \mathrm{~mL}$ of $1 \%$ potassium ferricyanide. The mixture was incubated at $50^{\circ} \mathrm{C}$ for $20 \mathrm{~min}$. Next, $2.5 \mathrm{~mL}$ of $10 \%$ trichloroacetic acid (w/v) was added. From this solution, $5 \mathrm{~mL}$ was mixed with $5 \mathrm{~mL}$ of distilled water and $1 \mathrm{~mL}$ of $0.1 \%$ ferric chloride and absorbance was measured spectrophotometrically at $700 \mathrm{~nm}$. BHA was used as standard.

2.3. Antimicrobial Activity. Series of novel indole analogues are tested for in vitro antimicrobial activity against gramnegative bacteria Escherichia coli ATCC 25922 and Klebsiella pneumoniae ATCC 33499 and gram-positive bacteria Staphylococcus aureus ATCC 6538 and antifungal activity against 
Candida tropicalis ATCC 8302 and Candida albicans ATCC 60193by applying the agar plate diffusion technique [33]. Dilution process was adopted at $25 \mu \mathrm{g}, 50 \mu \mathrm{g}$, and $100 \mu \mathrm{g} / \mathrm{mL}$ concentrations, respectively. The activity is compared with reference drugs gentamycin for antibacterial and fluconazole for antifungal activity. The zone of inhibition after $24 \mathrm{hr}$ of incubation at $37^{\circ} \mathrm{C}$ in case of antibacterial activity and $48 \mathrm{hr}$ in case of antifungal activity was compared with that of standards.

\section{Results and Discussion}

3.1. Chemistry. Molecules were designed with the aim of exploring their antioxidant and antimicrobial activities. The target compounds were synthesized as outlined in (Scheme 1). 3,5-Disubstitutedindole-2-carboxyhydrazides were reacted with carbon disulphide in the presence of base and hydrazine hydrate to get 5-(5-substituted-3-phenyl- $1 \mathrm{H}-$ indol-2-yl)-4-amino-4H-1,2,4-triazole-3-thiols 1a-c. Claisen-Schmidt condensation of 2,5-disubstituted indole-3carboxaldehydes with substituted acetophenones produced 3 -(2,5 - disubstituted-1H-indol-3-yl)-1-(4 - substituted - phenyl)prop-2-en-1-one 2a-i. The synthesized compounds $3 \mathbf{a}-\mathbf{z}$ were obtained in good yield by cyclocondensation of 5-(5-substituted-3-phenyl-1H-indol-2-yl)-4-amino- $4 \mathrm{H}$ 1,2,4-triazole-3-thiol la-c with 3-(2,5-disubstituted-1Hindol-3-yl)-1(4-substituted phenyl)prop-2-en-1-one $\mathbf{2 a - i}$. The formation of products was monitored by TLC. All the newly synthesized compounds were characterized by IR, ${ }^{1} \mathrm{H}$ NMR, ${ }^{13} \mathrm{C}$ NMR, mass spectroscopic and analytical data. The IR spectrum of 8-(5-chloro-2-phenyl-1H-indol-3-yl)-3(5-chloro-3-phenyl-1H-indol-2-yl)-6-(3-chlorophenyl)-[1, $2,4]$ triazolo[ $[3,4-\mathrm{b}][1,3,4]$ thiadiazepine $\mathbf{3 a}$ showed a strong absorption at $3180 \mathrm{~cm}^{-1}$ and $3090 \mathrm{~cm}^{-1}$ corresponding to indole $\mathrm{NH}$, absorption at 1654 and 1624 , corresponding to triazole $\mathrm{C}=\mathrm{N}$, and absorption at $1546 \mathrm{~cm}^{-1}$ corresponding to thiadiazepine $\mathrm{C}=\mathrm{N}$ stretching, respectively. The ${ }^{1} \mathrm{H}$ NMR spectrum of 3a has exhibited a singlet at $\delta 12.47 \mathrm{ppm}$ due to indole $\mathrm{NH}$ and peak at $\delta 11.63 \mathrm{ppm}$ is due to indole $\mathrm{NH}$ which is also $\mathrm{D}_{2} \mathrm{O}$ exchangeable. A multiplet between $\delta 7.31-8.47 \mathrm{ppm}$ corresponds to twenty aromatic protons present in the molecule and a peak at $\delta 5.65 \mathrm{ppm}$ is assigned for the $-\mathrm{CH}=$ of thiadiazepine ring proton. The ${ }^{13} \mathrm{C}$ NMR spectrum of compound 3a has shown peaks at $\delta 108,111$, $113,117,118,118,118,120,123,125,126,126,126,128,128,128$, $128,129,129,129,130,132,133,134,135,138,138,144,145$, and 166. The mass spectrum of compound 3a has shown molecular ion peak at $m / z 712[\mathrm{M}]^{+\bullet}$ which is corresponding to molecular weight of the compound. The above spectral data supports the formation of compound $\mathbf{3 a}$.

Various new triazolothiadiazepinylindole analogues synthesized during the present investigation are listed in (Table 1).

3.2. Biological Activities. The compounds $\mathbf{3 a}-\mathbf{z}$ were screened for their antioxidant (free radical scavenging, total antioxidant capacity, and ferric reducing antioxidant power) and antimicrobial activities.

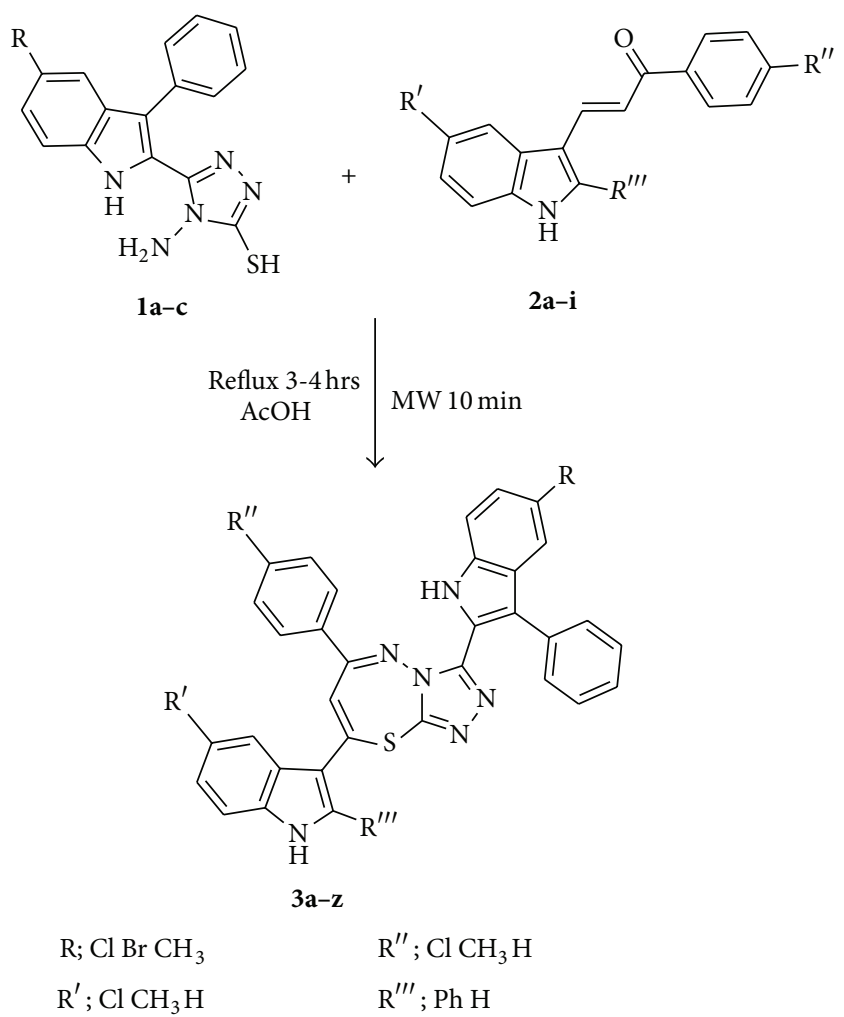

Scheme 1: Schematic representation for the formation of novel triazolothiadiazepinylindole $\mathbf{3 a}-\mathbf{z}$.

\subsubsection{Antioxidant Activities}

(1) Free Radical Scavenging Activity. The target compounds were screened for free radical scavenging activity by DPPH method [32]. The samples were prepared at concentrations of 25,50 , and $100 \mu \mathrm{g} / 100 \mu \mathrm{L}$ and butylated hydroxy anisole (BHA) was taken as standard. DPPH is a stable free radical in a methanolic solution. Because of the unpaired electron of $\mathrm{DPPH}$, it gives a strong absorption maxima at $517 \mathrm{~nm}$ in the visible region (purple color). In addition, the unpaired electron of the radical becomes paired in the presence of a hydrogen donor (a free radical scavenging antioxidant), decreasing the absorption. Among the compounds tested $\mathbf{3 a}-$ $\mathbf{c}$ and $\mathbf{3} \mathbf{j}-\mathbf{l}$ have shown very promising free radical scavenging activity. The increased activity is due to the existence of halogen substitution at the five positions of both indoles. The hydrogen of indole NH could be donated to the DPPH to form DPPH free radical; by the presence of phenyl ring at the third position of indole, the DPPH free radical will be stabilized by the resonance. Compounds $\mathbf{3 d}-\mathbf{f}, \mathbf{3} \mathbf{m}-$ $\mathbf{o}$, and $3 \mathbf{s}-\mathbf{x}$ containing halogen atom at five positions of indole and a methyl group at another indole ring have shown moderate activity, whereas compounds $3 \mathbf{g}-\mathbf{i}, \mathbf{3} \mathbf{p}-$ $\mathbf{r}$, and $3 \mathbf{y}-\mathbf{z}$ have shown the least activity compared with the standard. The bar graph representation of percentage of free radical scavenging activity is displayed in Figures 1 and 2. 
TABLE 1: Comparative data of conventional and microwave methods for the synthesis of novel triazolothiadiazepinylindole 3a-z.

\begin{tabular}{|c|c|c|c|c|c|c|c|c|c|}
\hline \multirow{2}{*}{$\begin{array}{l}\text { Compd }^{\mathrm{a}} \\
\text { Number }\end{array}$} & \multicolumn{4}{|c|}{ Substituents } & \multicolumn{2}{|c|}{ Conventional method } & \multicolumn{2}{|c|}{ Microwave method } & \multirow{2}{*}{$\begin{array}{l}\text { m.p. }{ }^{c} \\
\left({ }^{\circ} \mathrm{C}\right)\end{array}$} \\
\hline & $\mathrm{R}$ & $\mathrm{R}^{\prime}$ & $\mathrm{R}^{\prime \prime}$ & $\mathrm{R}^{\prime \prime \prime}$ & $\begin{array}{l}\text { Time } \\
\text { (min) }\end{array}$ & Yield $^{\mathrm{b}}(\%)$ & $\begin{array}{l}\text { Time } \\
(\mathrm{min})\end{array}$ & Yield $^{\mathrm{b}}(\%)$ & \\
\hline $3 a$ & $\mathrm{Cl}$ & $\mathrm{Cl}$ & $\mathrm{Cl}$ & $\mathrm{Ph}$ & $180-240$ & 85 & 10 & 95 & $200-02$ \\
\hline $3 b$ & $\mathrm{Cl}$ & $\mathrm{Cl}$ & $\mathrm{H}$ & $\mathrm{Ph}$ & $180-240$ & 80 & 10 & 93 & $142-43$ \\
\hline $3 c$ & $\mathrm{Cl}$ & $\mathrm{Cl}$ & $\mathrm{Me}$ & $\mathrm{Ph}$ & $180-240$ & 75 & 10 & 95 & $194-96$ \\
\hline $3 d$ & $\mathrm{Cl}$ & $\mathrm{Me}$ & $\mathrm{Cl}$ & $\mathrm{Ph}$ & $180-240$ & 80 & 10 & 95 & $160-62$ \\
\hline $3 e$ & $\mathrm{Cl}$ & $\mathrm{Me}$ & $\mathrm{H}$ & $\mathrm{Ph}$ & $180-240$ & 70 & 10 & 93 & $190-92$ \\
\hline $3 f$ & $\mathrm{Cl}$ & $\mathrm{Me}$ & $\mathrm{Me}$ & $\mathrm{Ph}$ & $180-240$ & 65 & 10 & 95 & $158-60$ \\
\hline $3 g$ & $\mathrm{Cl}$ & $\mathrm{H}$ & $\mathrm{Cl}$ & $\mathrm{H}$ & $180-240$ & 60 & 10 & 85 & $195-97$ \\
\hline $3 \mathrm{~h}$ & $\mathrm{Cl}$ & $\mathrm{H}$ & $\mathrm{H}$ & $\mathrm{H}$ & $180-240$ & 60 & 10 & 80 & $168-70$ \\
\hline $3 \mathbf{i}$ & $\mathrm{Cl}$ & $\mathrm{H}$ & $\mathrm{Me}$ & $\mathrm{H}$ & $180-240$ & 70 & 10 & 90 & $155-57$ \\
\hline $3 \mathbf{j}$ & $\mathrm{Br}$ & $\mathrm{Cl}$ & $\mathrm{Cl}$ & $\mathrm{Ph}$ & $180-240$ & 80 & 10 & 98 & $210-12$ \\
\hline $3 \mathbf{k}$ & $\mathrm{Br}$ & $\mathrm{Cl}$ & $\mathrm{H}$ & $\mathrm{Ph}$ & $180-240$ & 85 & 10 & 96 & $195-97$ \\
\hline 31 & $\mathrm{Br}$ & $\mathrm{Cl}$ & $\mathrm{Me}$ & $\mathrm{Ph}$ & $180-240$ & 85 & 10 & 95 & $140-42$ \\
\hline $3 m$ & $\mathrm{Br}$ & $\mathrm{Me}$ & $\mathrm{Cl}$ & $\mathrm{Ph}$ & $180-240$ & 75 & 10 & 90 & $180-82$ \\
\hline $3 n$ & $\mathrm{Br}$ & $\mathrm{Me}$ & $\mathrm{H}$ & $\mathrm{Ph}$ & $180-240$ & 65 & 10 & 85 & $165-67$ \\
\hline 30 & $\mathrm{Br}$ & $\mathrm{Me}$ & $\mathrm{Me}$ & $\mathrm{Ph}$ & $180-240$ & 60 & 10 & 80 & $168-70$ \\
\hline $3 p$ & $\mathrm{Br}$ & $\mathrm{H}$ & $\mathrm{Cl}$ & $\mathrm{H}$ & $180-240$ & 60 & 10 & 85 & $210-12$ \\
\hline $3 q$ & $\mathrm{Br}$ & $\mathrm{H}$ & $\mathrm{H}$ & $\mathrm{H}$ & $180-240$ & 60 & 10 & 75 & $218-20$ \\
\hline $3 r$ & $\mathrm{Br}$ & $\mathrm{H}$ & $\mathrm{Me}$ & $\mathrm{H}$ & $180-240$ & 60 & 10 & 80 & $120-22$ \\
\hline $3 s$ & $\mathrm{Me}$ & $\mathrm{Cl}$ & $\mathrm{Cl}$ & $\mathrm{Ph}$ & $180-240$ & 75 & 10 & 85 & $183-85$ \\
\hline $3 t$ & $\mathrm{Me}$ & $\mathrm{Cl}$ & $\mathrm{H}$ & $\mathrm{Ph}$ & $180-240$ & 75 & 10 & 85 & 201-02 \\
\hline $3 \mathbf{u}$ & $\mathrm{Me}$ & $\mathrm{Cl}$ & $\mathrm{Me}$ & $\mathrm{Ph}$ & $180-240$ & 80 & 10 & 87 & $181-83$ \\
\hline $3 v$ & $\mathrm{Me}$ & $\mathrm{Me}$ & $\mathrm{Cl}$ & $\mathrm{Ph}$ & $180-240$ & 65 & 10 & 85 & $190-92$ \\
\hline $3 w$ & $\mathrm{Me}$ & $\mathrm{Me}$ & $\mathrm{H}$ & $\mathrm{Ph}$ & $180-240$ & 60 & 10 & 80 & $161-62$ \\
\hline $3 x$ & $\mathrm{Me}$ & $\mathrm{Me}$ & $\mathrm{Me}$ & $\mathrm{Ph}$ & $180-240$ & 65 & 10 & 86 & $172-74$ \\
\hline $3 y$ & $\mathrm{Me}$ & $\mathrm{H}$ & $\mathrm{H}$ & $\mathrm{H}$ & $180-240$ & 60 & 10 & 75 & $158-60$ \\
\hline $3 z$ & $\mathrm{Me}$ & $\mathrm{H}$ & $\mathrm{Cl}$ & $\mathrm{H}$ & $180-240$ & 60 & 10 & 70 & $149-51$ \\
\hline
\end{tabular}

${ }^{\mathrm{a}}$ Products were characterized by IR, ${ }^{1} \mathrm{H}$ NMR, ${ }^{13} \mathrm{C}$ NMR, MS, and elemental analysis. ${ }^{\mathrm{b}}$ Isolated yield. ${ }^{\mathrm{c}}$ Melting points are uncorrected.

(2) Total Antioxidant Capacity. Total antioxidant activity was performed to all the newly synthesized compounds [34]. Antioxidant capacities are expressed as equivalents of ascorbic acid. Among the tested compounds $\mathbf{3 a}-\mathbf{c}$ and $\mathbf{3 j}-$ $\mathbf{l}$ which are halogen substituted triazolothiadiazepinylindole have shown very strong total antioxidant capacity. Compounds with methyl substitution at the fifth position of the indole ring and no substitution at the second and fifth positions have shown the least total antioxidant capacity compared with the standard. The increased activity is due to the presence of halogen at the fifth position and a phenyl ring at the third position of indole. The results of total antioxidant activity are shown in Figures 3 and 4.

(3) Ferric Reducing Antioxidant Power Activity. The novel compounds were screened for ferric reducing antioxidant activity [35]. Butylated hydroxy anisole (BHA) was used as standard. All the tested compounds have shown positive tendency towards the ferric reducing activity. The presence of reducer (i.e., antioxidant) causes the reduction of the $\mathrm{Fe}^{+3}$ /ferricyanide complex to the $\mathrm{Fe}^{+2}$ form after the addition of trichloroacetic acid and ferric chloride. The reducing power of test compounds increases with increase in concentration. Compounds $\mathbf{3 d}-\mathbf{f}, \mathbf{3 m - \mathbf { m }}$, and $\mathbf{3 s}-\mathbf{z}$ have shown excellent ferric reducing antioxidant activity and other analogues of indole have shown moderate to high activity. The presence of methyl group at the fifth position of the indole ring plays an important role as a better electron donor which enhances reducing power activity of the compounds. The results are presented in Figures 5 and 6.

3.3. Antimicrobial Activity. Applying the agar plate diffusion technique [33], series of novel triazolothiadiazepinylindole 
TABLE 2: Zone of inhibition in $\mathrm{mm}$ at 25,50 , and $100 \mu \mathrm{g} / \mathrm{mL}$ concentrations.

\begin{tabular}{|c|c|c|c|c|c|c|c|c|c|c|c|c|c|c|c|}
\hline \multirow{3}{*}{ Compd name } & \multicolumn{9}{|c|}{ Antibacterial activity } & \multicolumn{6}{|c|}{ Antifungal activity } \\
\hline & \multicolumn{3}{|c|}{ S. aureus } & \multicolumn{3}{|c|}{ E. coli } & \multicolumn{3}{|c|}{ K. pneumoniae } & \multicolumn{3}{|c|}{ C. tropicalis } & \multicolumn{3}{|c|}{ C. albicans } \\
\hline & 25 & 50 & 100 & 25 & 50 & 100 & 25 & 50 & 100 & 25 & 50 & 100 & 25 & 50 & 100 \\
\hline $3 \mathbf{a}$ & 13 & 17 & 20 & 15 & 20 & 22 & 16 & 22 & 25 & 14 & 18 & 20 & 13 & 15 & 18 \\
\hline $3 b$ & 14 & 16 & 20 & 16 & 19 & 24 & 15 & 21 & 24 & 15 & 17 & 21 & 14 & 17 & 19 \\
\hline $3 c$ & 15 & 15 & 19 & 14 & 21 & 25 & 14 & 23 & 26 & 13 & 16 & 20 & 15 & 16 & 20 \\
\hline $3 d$ & 11 & 12 & 17 & 12 & 15 & 16 & 09 & 14 & 15 & 10 & 12 & 15 & 10 & 12 & 14 \\
\hline $3 e$ & 10 & 13 & 15 & 11 & 16 & 17 & 08 & 15 & 18 & 09 & 11 & 14 & 09 & 11 & 12 \\
\hline $3 f$ & 09 & 12 & 16 & 09 & 14 & 16 & 09 & 13 & 14 & 09 & 12 & 13 & 09 & 10 & 10 \\
\hline $3 g$ & 02 & 06 & 08 & 05 & 07 & 08 & 05 & 07 & 09 & 02 & 04 & 06 & 03 & 04 & 05 \\
\hline $3 \mathrm{~h}$ & 03 & 04 & 09 & 03 & 05 & 07 & 04 & 08 & 10 & 01 & 03 & 05 & 03 & 06 & 09 \\
\hline $3 \mathbf{i}$ & 05 & 07 & 08 & 04 & 06 & 08 & 03 & 06 & 08 & 03 & 04 & 06 & 05 & 06 & 08 \\
\hline $3 \mathbf{j}$ & 14 & 18 & 21 & 18 & 18 & 23 & 15 & 21 & 23 & 15 & 18 & 21 & 13 & 15 & 17 \\
\hline $3 \mathbf{k}$ & 13 & 19 & 20 & 17 & 20 & 22 & 17 & 23 & 24 & 16 & 19 & 21 & 15 & 17 & 18 \\
\hline 31 & 12 & 18 & 21 & 16 & 19 & 25 & 16 & 22 & 26 & 12 & 15 & 21 & 13 & 16 & 19 \\
\hline $3 m$ & 10 & 10 & 15 & 10 & 12 & 15 & 09 & 15 & 16 & 10 & 12 & 15 & 09 & 11 & 12 \\
\hline $3 n$ & 08 & 11 & 14 & 08 & 13 & 14 & 10 & 13 & 18 & 09 & 11 & 14 & 09 & 09 & 11 \\
\hline 30 & 08 & 10 & 14 & 09 & 14 & 16 & 09 & 13 & 19 & 08 & 10 & 12 & 10 & 10 & 12 \\
\hline $3 p$ & 03 & 04 & 09 & 04 & 05 & 08 & 06 & 08 & 12 & 04 & 06 & 08 & 03 & 05 & 07 \\
\hline $3 q$ & 03 & 05 & 07 & 03 & 05 & 08 & 05 & 09 & 11 & 03 & 05 & 07 & 05 & 06 & 08 \\
\hline $3 \mathbf{r}$ & 04 & 06 & 08 & 06 & 08 & 09 & 04 & 08 & 10 & 02 & 05 & 06 & 03 & 06 & 07 \\
\hline $3 s$ & 10 & 10 & 15 & 10 & 12 & 15 & 09 & 14 & 15 & 10 & 12 & 14 & 11 & 12 & 13 \\
\hline $3 t$ & 09 & 11 & 13 & 11 & 14 & 17 & 10 & 15 & 18 & 08 & 11 & 15 & 09 & 10 & 12 \\
\hline $3 \mathbf{u}$ & 08 & 11 & 14 & 08 & 13 & 14 & 10 & 13 & 14 & 09 & 13 & 13 & 08 & 12 & 14 \\
\hline $3 v$ & 09 & 09 & 16 & 10 & 12 & 15 & 11 & 12 & 17 & 10 & 12 & 15 & 09 & 13 & 15 \\
\hline $3 w$ & 08 & 10 & 14 & 09 & 14 & 16 & 09 & 12 & 15 & 09 & 11 & 16 & 10 & 11 & 12 \\
\hline $3 x$ & 09 & 12 & 12 & 09 & 11 & 14 & 08 & 14 & 16 & 08 & 10 & 17 & 09 & 10 & 12 \\
\hline $3 y$ & 04 & 06 & 09 & 05 & 08 & 10 & 05 & 09 & 10 & 05 & 09 & 10 & 04 & 05 & 06 \\
\hline $3 z$ & 05 & 05 & 08 & 04 & 09 & 11 & 04 & 10 & 12 & 04 & 08 & 11 & 04 & 04 & 05 \\
\hline Std.1 & 15 & 19 & 22 & 18 & 21 & 25 & 17 & 23 & 27 & - & - & - & - & - & - \\
\hline Std.2 & - & - & - & - & - & - & - & - & - & 15 & 19 & 22 & 16 & 19 & 21 \\
\hline
\end{tabular}

Std.1: gentamycin, Std.2: fluconazole.

The bold font refers to the compounds which have shown more potent antimicrobial activities.

analogues were screened for in vitro antibacterial activity against (Table 2) gram-negative bacteria Escherichia coli (E. coli) and Klebsiella pneumoniae (K. pneumoniae) and gram-positive bacteria Staphylococcus aureus (S. aureus) at $25 \mu \mathrm{g} / \mathrm{mL}, 50 \mu \mathrm{g} / \mathrm{mL}$, and $100 \mu \mathrm{g} / \mathrm{mL}$ concentrations, respectively. Gentamycin was used as standard. The zone of inhibitions was measured in $\mathrm{mm}$ for each concentration. Most of the screened compounds were found to have significant antibacterial activity. Compounds $\mathbf{3} \mathbf{a}-\mathbf{c}$ and $\mathbf{3 j} \mathbf{j}-\mathbf{l}$ have shown very good activity against all the three bacterial strains. Compounds $\mathbf{3 d}-\mathbf{f}, \mathbf{3 m}-\mathbf{o}$, and $\mathbf{3 s}-\mathbf{x}$ have shown moderate activity and compounds $\mathbf{3 g}-\mathbf{i}, \mathbf{3 p}-\mathbf{r}$, and $\mathbf{3 y}-\mathbf{z}$ have shown the least activity. Antifungal screening of the compounds was carried out in vitro against two fungi strains Candida tropicalis and Candida albicans at $25 \mu \mathrm{g} / \mathrm{mL}, 50 \mu \mathrm{g} / \mathrm{mL}$, and $100 \mu \mathrm{g} / \mathrm{mL}$ concentrations using fluconazole as standard. Among the tested indole analogues the majority of compounds exhibited moderate to significant antifungal activity.

\section{Conclusions}

We have synthesized titled compounds $\mathbf{3 a}-\mathbf{z}$ by economic, better yield, and safer methods through the formation of compounds $\mathbf{1 a}-\mathbf{c}$ and $\mathbf{2 a}-\mathbf{i}$ under thermal and microwave condition. The compounds $\mathbf{3 a}-\mathbf{z}$ were subjected for their antioxidant and antimicrobial screening. Very potent antimicrobial, scavenging and antioxidant activity was observed with compounds containing halogens at the fifth position of indoles. Excellent ferric reducing activity was observed with compounds containing electron donor group at five positions 


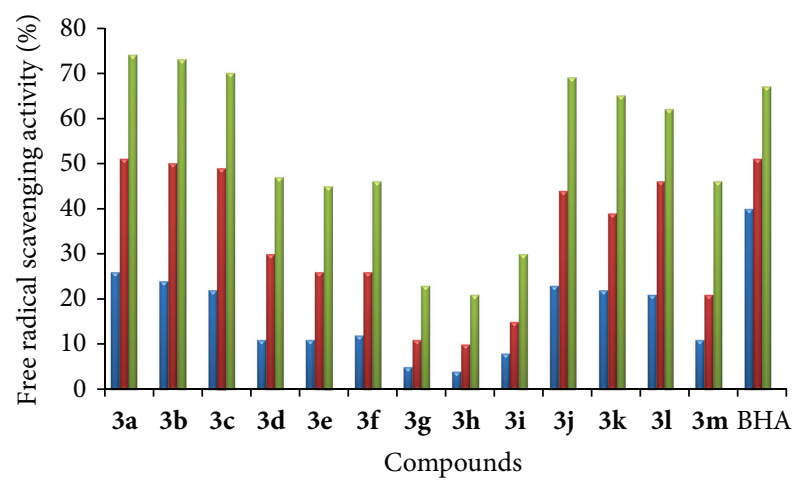

- $25 \mu \mathrm{g}$

- $50 \mu \mathrm{g}$

$\square 100 \mu \mathrm{g}$

FIGURE 1: Free radical scavenging activity of $\mathbf{3 a - m}$.

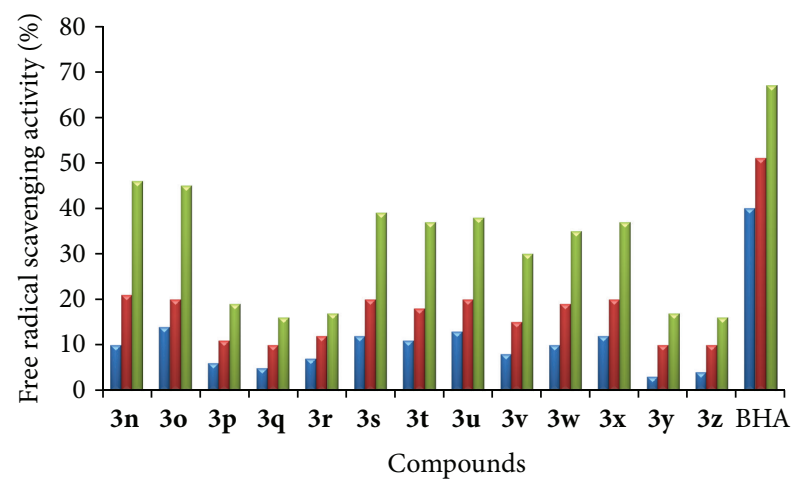

๑ $25 \mu \mathrm{g}$

․ $50 \mu \mathrm{g}$

$\square 100 \mu \mathrm{g}$

FIGURE 2: Free radical scavenging activity of $3 \mathbf{n}-\mathbf{z}$.

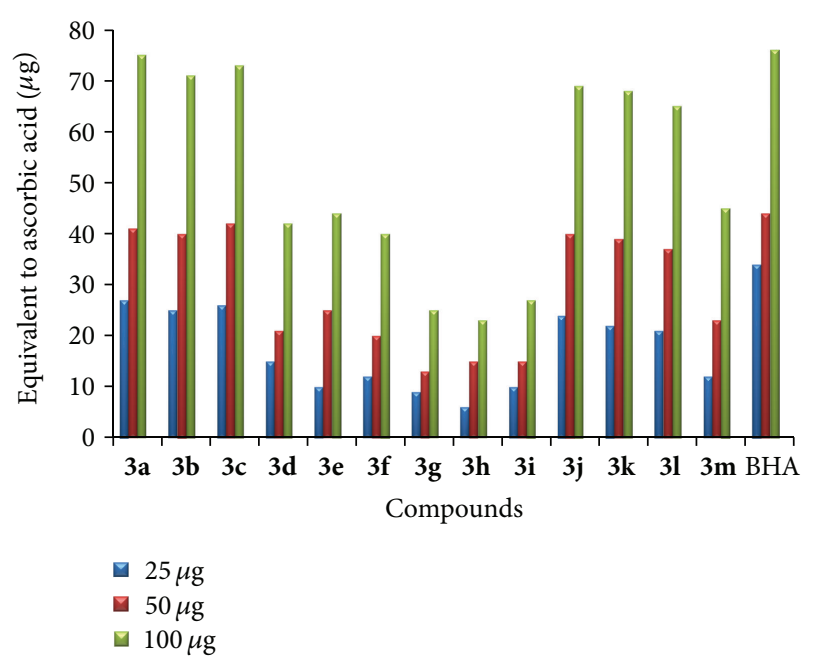

FIgURE 3: Total antioxidant capacity of $\mathbf{3 a} \mathbf{a} \mathbf{m}$.

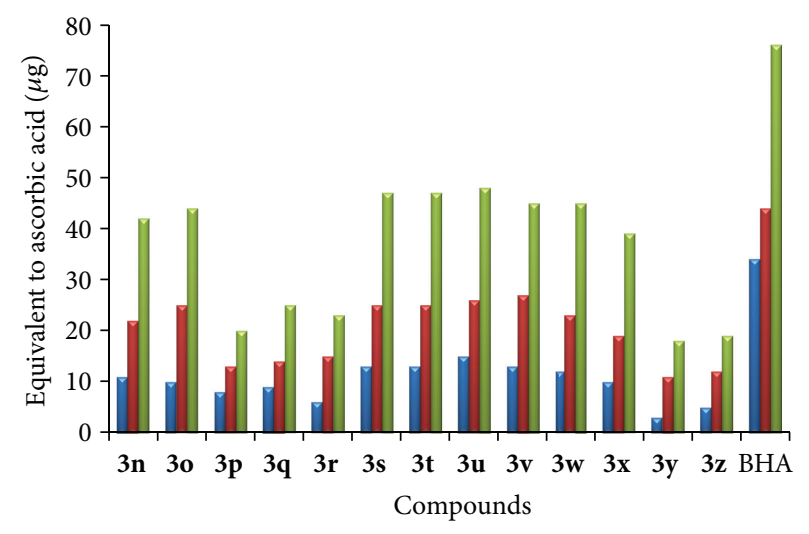

ㅁ $25 \mu \mathrm{g}$

$50 \mu \mathrm{g}$

$\square 100 \mu \mathrm{g}$

FIgURE 4: Total antioxidant capacity of $\mathbf{3 n}-\mathbf{z}$.

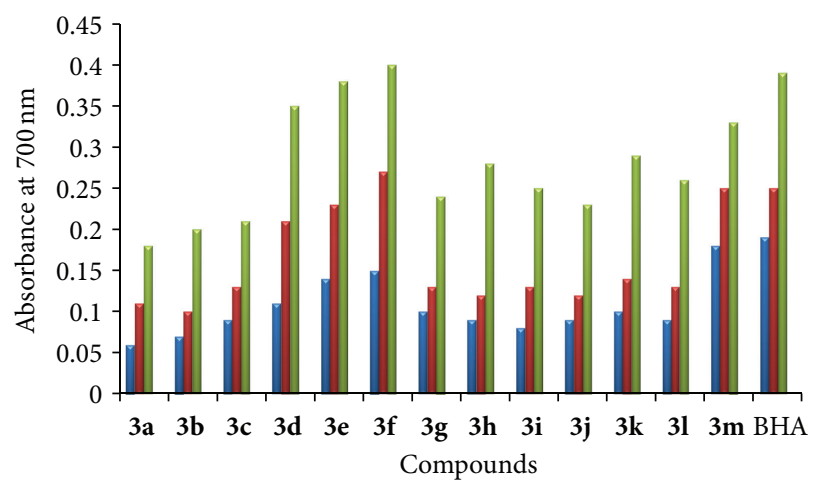

\ $25 \mu \mathrm{g}$

$\square 50 \mu \mathrm{g}$

$\square 100 \mu \mathrm{g}$

FIGURE 5: Ferric reducing antioxidant power activity of $\mathbf{3 a - m}$.

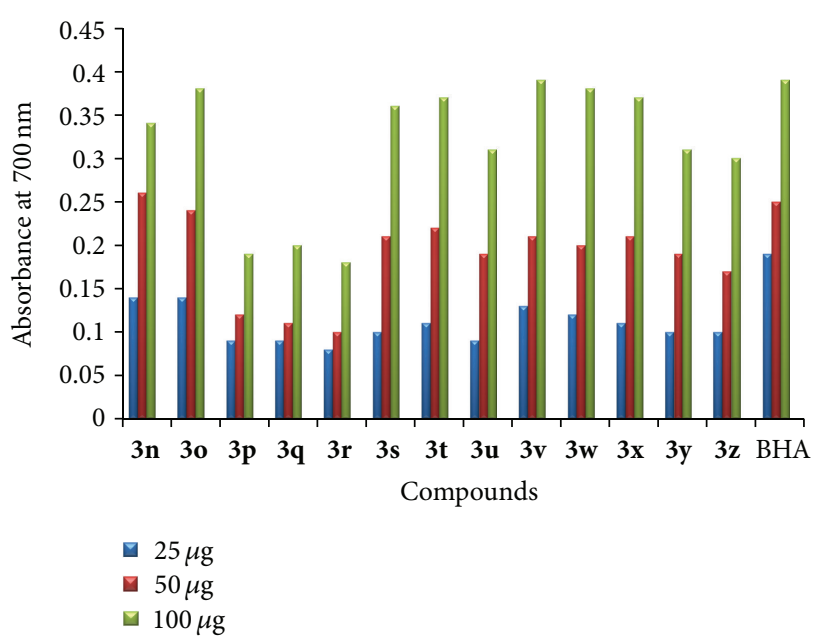

FIGURE 6: Ferric reducing antioxidant power activity of $\mathbf{3 n}-\mathbf{z}$. 
of one/both indoles. Therefore, the findings will provide a great impact on chemists and biochemists for further investigations in the indole field in search of molecules possessing potent antioxidant and antimicrobial activities.

\section{Conflict of Interests}

The authors declare that there is no conflict of interests regarding the publication of this paper.

\section{Acknowledgments}

The authors thank the Department of Chemistry, Gulbarga University, Gulbarga. The authors are also thankful to the director of IISc (Bangalore) and IIT (Madras) for spectroscopic analysis. They also extend their sincere thanks to BIOGENICS, Hubli, and Department of Microbiology, Gulbarga University, Gulbarga, for their assistance in carrying out biological activities.

\section{References}

[1] S. V. Lennon, S. J. Martin, and T. G. Cotter, "Dose-dependent induction of apoptosis in human tumour cell lines by widely diverging stimuli," Cell Proliferation, vol. 24, no. 2, pp. 203-214, 1991.

[2] H. Shirinzadeh, B. Eren, H. Gurer-Orhan, S. Suzen, and S. Özden, "Novel indole-based analogs of melatonin: synthesis and in vitro antioxidant activity studies," Molecules, vol. 15, no. 4, pp. 2187-2202, 2010.

[3] M. L. Circu and T. Y. Aw, "Reactive oxygen species, cellular redox systems, and apoptosis," Free Radical Biology and Medicine, vol. 48, no. 6, pp. 749-762, 2010.

[4] Y. Hangun-Balkir and M. L. McKenney, "Determination of antioxidant activities of berries and resveratrol," Green Chemistry Letters and Reviews, vol. 5, no. 2, pp. 147-153, 2012.

[5] F. Regoli and G. Principato, "Glutathione, glutathionedependent and antioxidant enzymes in mussel, Mytilus galloprovincialis exposed to metals under field and laboratory conditions: implications for the use of biochemical biomarkers," Aquatic Toxicology, vol. 31, no. 2, pp. 143-164, 1995.

[6] N. Topalca, E. Yegin, and I. Celik, "Influence of indole-3butyric acid on antioxidant defense systems in various tissues of rats at subacute and subchronic exposure," Food and Chemical Toxicology, vol. 47, no. 10, pp. 2441-2444, 2009.

[7] H. E. Poulsen, H. Prieme, and S. Loft, "Role of oxidative DNA damage in cancer initiation and promotion," European Journal of Cancer Prevention, vol. 7, no. 1, pp. 9-16, 1998.

[8] M. Liu, X. Q. Li, C. Weber, C. Y. Lee, J. Brown, and R. H. Liu, "Antioxidant and antiproliferative activities of raspberries," Journal of Agricultural and Food Chemistry, vol. 50, no. 10, pp. 2926-2930, 2002.

[9] B. Halliwell, "Role of free radicals in the neurodegenerative diseases: therapeutic implications for antioxidant treatment," Drugs \& Aging, vol. 18, no. 9, pp. 685-716, 2001.

[10] K. Sudha, A. Rao, S. Rao, and A. Rao, "Free radical toxicity and antioxidants in Parkinson's disease," Neurology India, vol. 51, no. 1, pp. 60-62, 2003.

[11] G. S. Yossi, M. Eldad, and O. Daniel, "Oxidative stress inducedneurodegenerative diseases: the need for antioxidants that penetrate the blood brain barrier," Neuropharmacology, vol. 40, no. 8, pp. 959-975, 2001.

[12] K. Asplund, "Antioxidant vitamins in the prevention of cardiovascular disease: a systematic review," Journal of Internal Medicine, vol. 251, no. 5, pp. 372-392, 2002.

[13] K. N. Prasad, W. C. Cole, and B. Kumar, "Multiple antioxidants in the prevention and treatment of Parkinson's disease," Journal of the American College of Nutrition, vol. 18, no. 5, pp. 413-423, 1999.

[14] C. Li-Hsun, C. Chia-Mao, B. S. Deepak, and S. ChungMing, "Divergent synthesis of unsymmetrical annulated biheterocyclic compound libraries: benzimidazole linked indolobenzodiazepines/quinoxaline," ACS Combinatorial Science, vol. 13, no. 4, pp. 391-398, 2011.

[15] M. García-Valverde and T. Torroba, "Special issue: sulfurnitrogen heterocycles," Molecules, vol. 10, no. 2, pp. 318-320, 2005.

[16] T. Karabasanagouda, A. V. Adhikari, and N. S. Shetty, "Synthesis and antimicrobial activities of some novel 1,2,4-triazolo[3,4-b]1,3,4-thiadiazoles and 1,2,4-triazolo[3,4-b]-1,3,4-thiadiazines carrying thioalkyl and sulphonyl phenoxy moieties," European Journal of Medicinal Chemistry, vol. 42, no. 4, pp. 521-529, 2007.

[17] M. D. Mullican, M. W. Wilson, D. T. Connor, C. R. Kostlan, D. J. Schrier, and R. D. Dyer, "Design of 5-(3,5-di-tert-butyl4-hydroxyphenyl)-1,3,4-thiadiazoles, -1,3,4-oxadiazoles, and 1,2,4-triazoles as orally-active, nonulcerogenic antiinflammatory agents," Journal of Medicinal Chemistry, vol. 36, no. 8, pp. 1090-1099, 1993.

[18] T. Wen-Jie and H. Yong-Zhou, "Simple and efficient one-pot synthesis of 2,4-diaryl-1,2,3-triazoles," Synthetic Communications, vol. 36, no. 17, pp. 2461-2468, 2006.

[19] Y. A. Al-Soud, N. A. Al-Masoudi, and A. R. Ferwanah, "Synthesis and properties of new substituted 1,2,4-triazoles: potential antitumor agents," Bioorganic \& Medicinal Chemistry, vol. 11, no. 8, pp. 1701-1708, 2003.

[20] B. S. Holla, B. Veerendra, M. K. Shivananda, and B. Poojary, "Synthesis characterization and anticancer activity studies on some Mannich bases derived from 1,2,4-triazoles," European Journal of Medicinal Chemistry, vol. 38, no. 7-8, pp. 759-767, 2003.

[21] S. F. Barbuceanu, G. L. Almajan, I. Saramet, C. Draghici, R. Socoteanu, and F. Barbuceanu, "New S-alkylated 1,2,4triazoles incorporating diphenyl sulfone moieties with potential antibacterial activity," Journal of the Serbian Chemical Society, vol. 74, no. 10, pp. 1041-1049, 2009.

[22] A.-R. Farghaly, E. De Clercq, and H. El-Kashef, "Synthesis and antiviral activity of novel $[1,2,4]$ triazolo[3,4-b] $[1,3,4]$ thiadiazoles, $[1,2,4]$ triazolo[3,4-b][1,3,4] thiadiazines and $[1,2,4]$ triazolo[3,4-b] [1,3,4] thiadiazepines," Arkivoc, vol. 2006, no. 10, pp. 137-151, 2006.

[23] M. Kidwai, P. Sapra, P. Misra, R. Saxena, and M. Singh, "Microwave assisted solid support synthesis of novel 1,2,4triazolo[3,4-b]-1,3,4-thiadiazepines as potent antimicrobial agents," Bioorganic \& Medicinal Chemistry, vol. 9, no. 2, pp. 217-220, 2001.

[24] E. R. Abbey, L. N. Zakharov, and S.-Y. Liu, "Boron in disguise: the parent fused BN indole," Journal of the American Chemical Society, vol. 133, no. 30, pp. 11508-11511, 2011.

[25] F.-R. Alexandre, A. Amador, S. Bot et al., "Synthesis and biological evaluation of aryl-phospho-indole as novel HIV1 non-nucleoside reverse transcriptase inhibitors," Journal of Medicinal Chemistry, vol. 54, no. 1, pp. 392-395, 2011. 
[26] A. J. Kochanowska-Karamyan and M. T. Hamann, "Marine indole alkaloids: potential new drug leads for the control of depression and anxiety," Chemical Reviews, vol. 110, no. 8, pp. 4489-4497, 2010.

[27] B. S. Sasidhar and J. S. Biradar, "Synthesis of some bisindolyl analogs for in vitro cytotoxic and DNA cleavage studies," Medicinal Chemistry Research, vol. 22, no. 7, pp. 3518-3526, 2013.

[28] J. S. Biradar and B. S. Sasidhar, "Solvent-free, microwave assisted Knoevenagel condensation of novel 2,5-disubstituted indole analogues and their biological evaluation," European Journal of Medicinal Chemistry, vol. 46, no. 12, pp. 6112-6118, 2011.

[29] J. S. Biradar, B. S. Sasidhar, and R. Parveen, "Synthesis, antioxidant and DNA cleavage activities of novel indole derivatives," European Journal of Medicinal Chemistry, vol. 45, no. 9, pp. 4074-4078, 2010.

[30] J. S. Biradar, R. Parveen, B. S. Sasidhar, and S. M. Praveen, "Synthesis of new indolyl benzodiazepines and their DNA cleavage and antimicrobial activities," Indian Journal of Heterocyclic Chemistry, vol. 20, pp. 181-182, 2010.

[31] J. S. Biradar, Studies in the indole field [Ph.D. thesis], Gulbarga University, Gulbarga, India, 1982.

[32] R. P. Singh, K. N. C. Murthy, and G. K. Jayaprakasha, "Studies on the antioxidant activity of pomegranate (Punica granatum) peel and seed extracts using in vitro models," Journal of Agricultural and Food Chemistry, vol. 50, no. 1, pp. 81-86, 2002.

[33] C. Praveen, A. Ayyanar, and P. T. Perumal, "Practical synthesis, anticonvulsant, and antimicrobial activity of $N$-allyl and $N$ propargyl di(indolyl)indolin-2-ones," Bioorganic \& Medicinal Chemistry Letters, vol. 21, no. 13, pp. 4072-4077, 2011.

[34] K. Mruthunjaya and V. I. Hukkeri, "In vitro antioxidant and free radical scavenging potential of Parkinsonia aculeata Linn," Pharmacognosy Magazine, vol. 4, no. 13, pp. 42-51, 2008.

[35] J. C. M. Barreira, I. C. F. R. Ferreira, M. B. P. P. Oliveira, and J. A. Pereira, "Antioxidant activity and bioactive compounds of ten Portuguese regional and commercial almond cultivars," Food and Chemical Toxicology, vol. 46, no. 6, pp. 2230-2235, 2008. 

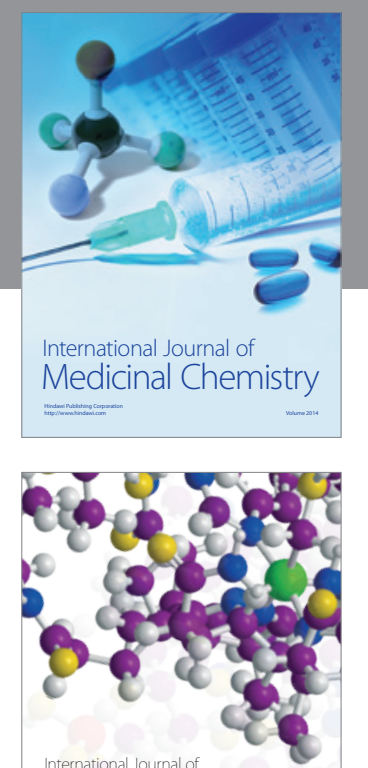

\section{Carbohydrate} Chemistry

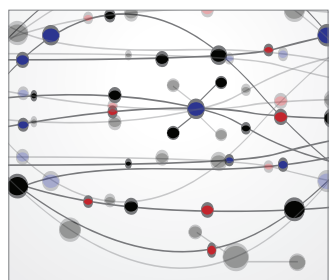

The Scientific World Journal
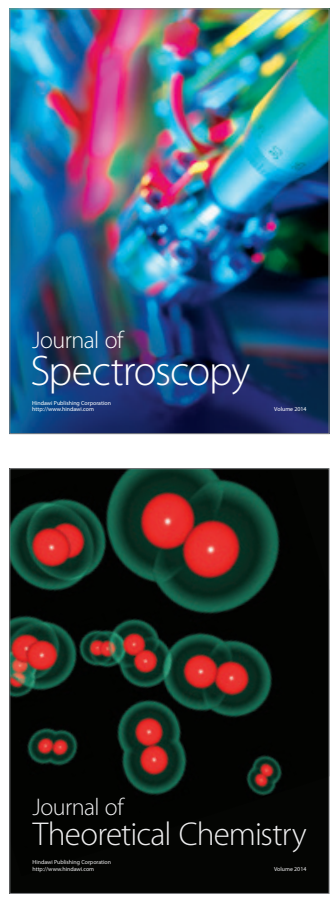
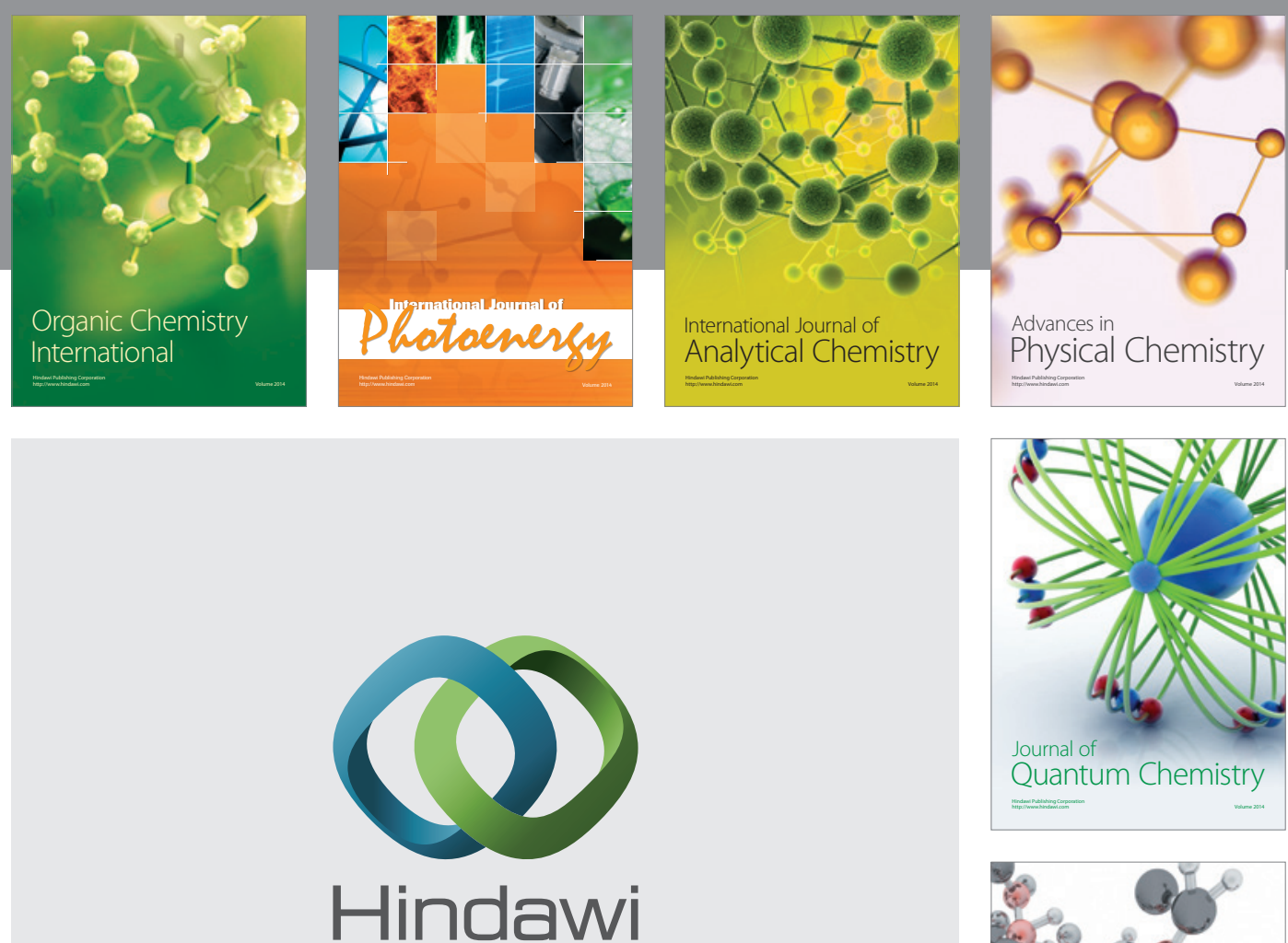

Submit your manuscripts at

http://www.hindawi.com

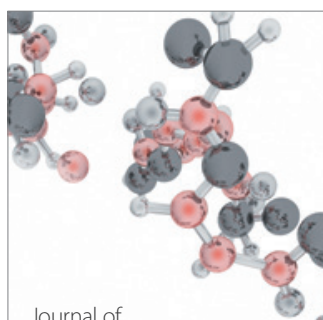

Analytical Methods

in Chemistry

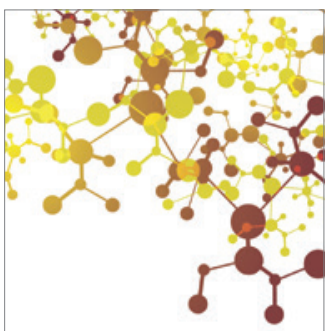

Journal of

Applied Chemistry

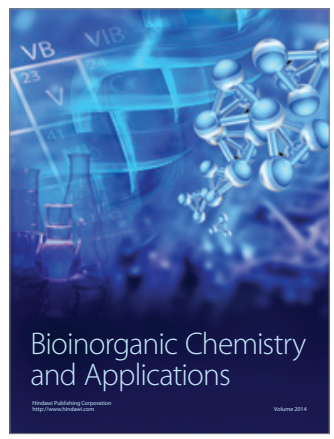

Inorganic Chemistry
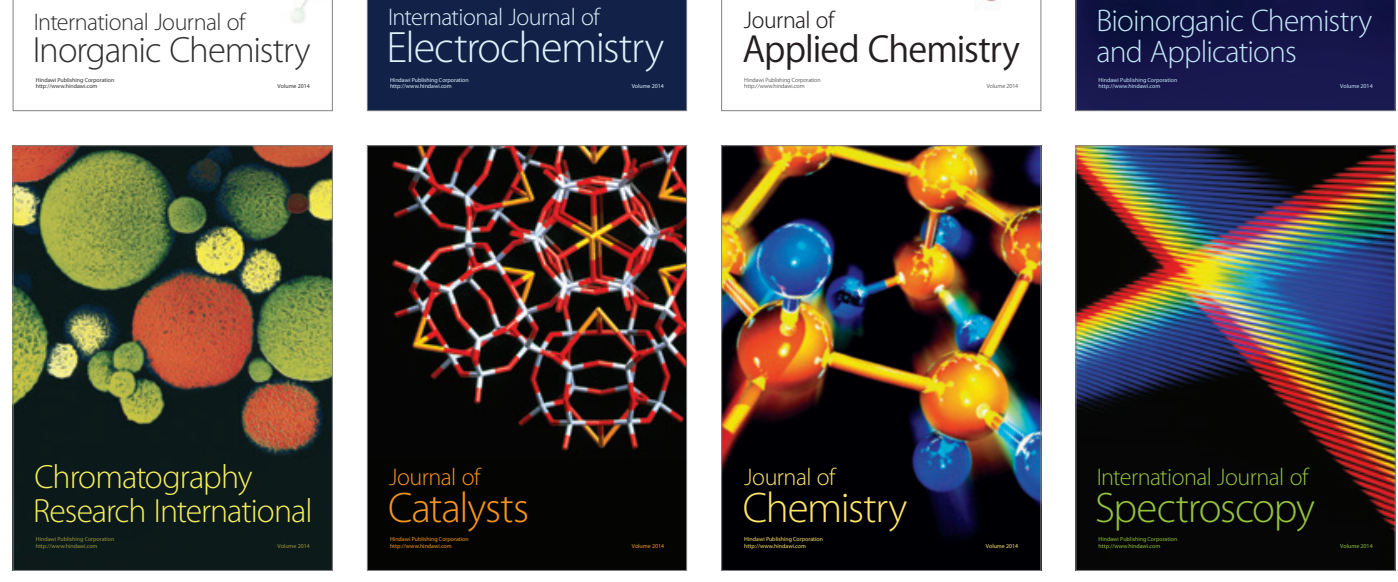